\title{
Proceedings of the 26th International Stroke Genetics Consortium Workshop: St. Louis, MO
}

Neurol Genet 2020;6:e422. doi:10.1212/NXG.0000000000000422

\section{Proceedings of the 26th International Stroke Genetics Consortium Workshop, November 2-4, 2019, St. Louis, MO, USA}

Jin-Moo Lee, $M D, P h D^{1}$, and Carlos Cruchaga, $P h D^{2}$

${ }^{1}$ Department of Neurology, Washington University School of Medicine; and ${ }^{2}$ Department of Psychiatry, Washington University School of Medicine, St. Louis, MO.

The 26th workshop of the International Stroke Genetics Consortium (ISGC) was held at the Four Seasons Hotel on November 2-4, 2019 in St. Louis, MO, and was hosted by Jin-Moo Lee, $\mathrm{MD}, \mathrm{PhD}$ and Carlos Cruchaga, $\mathrm{PhD}$, and co-hosted by Stephanie Debette, MD, PhD. The ISGC is an international collaboration of physicians and scientists who have agreed to pool resources and expertise in an effort to unravel the genetic basis of stroke and its comorbidities. Founded in 2007 by a small group of stroke genetics investigators, the ISGC has grown to over 200 members representing over 50 countries in North and South America, Europe, Australia, Africa, and Asia. ISGC workshops are held semi-annually and provide a forum for ISGC investigators to report on progress of ongoing scientific projects and discuss new ideas that help advance the ISGC's research mission. The ISGC nurtures junior investigators by offering several travel awards to young investigators interested in stroke genetics. Many abstracts in these Proceedings reflect the contributions of active junior investigators. Herein, we present the Proceedings and official published abstracts of the 26th ISGC Workshop.

Presentations at the workshop included invited lectures, ISGC working group reports, and short presentations about ongoing research projects and new collaborative proposals. With the themed title of "Big Data Science: Genomes, Epigenomes, Microbiomes, and Connectomes," invited speakers included Dr. Jeffrey Gordon, "Grandfather of the Microbiome”; Dr. David Van Essen, PI of the Human Connectome Project; Dr. Ting Wang, one of world leaders in human epigenomics; and Dr. Christina Gurnett, an expert in the genetic basis of developmental neurological disorders.

The ISGC actively invites other like-minded collaborative scientists to join the consortium. The next Workshops will be held in Bordeaux, France in May 2020, and in Melbourne, Australia in October 2020. We encourage you to visit our website (http://www.strokegenetics.org) for further details.

On behalf of the ISGC Steering Committee and its many members, we hope that you enjoy reviewing these Proceedings.

Full author information, including institutions, disclosures, and contact information, can be found at: links.lww.com/NXG/A259.

Acknowledgment: Steering Committee-Jin-Moo Lee, Chair and Israel Fernandez-Cadenas, Co-Chair; Members: Stephanie Debette (Immediate Past-Chair), Ann-Katrin Giese (Junior member); Rufus Akinyemi, Jemma Hopewell, Steven Kittner, Jane Maguire, Paul Nyquist, Natalia Rost. Working Group Leaders-Acute Endophenotypes WG: Israel Fernandez-Cadenas, Jin-Moo Lee; Cognitive WG: Matt Pase, Brad Worrall; Imaging WG: Natalia Rost; Intracranial Aneurysm WG: Ynte Ruigrok, Philippe Bijlenga; Intracerebral Hemorrhage WG: Guido Falcone, Jonathan Rosand, Dan Woo; Meta/MegaStroke WG: Stephanie Debette, Martin Dichgans, Jemma 
Hopewell; Mutliomics WG: Carlos Cruchaga, Myriam Fornage; Neuro-CHARGE WG: Myriam Fornage, Sudha Seshadri; SiGN WG: Steven Kittner, Brackie Mitchel; Translational Science WG: Chris Anderson, Tom Van Agtmael.

Correspondence Dr. Lee leejm@wustl.edu

\section{Quantitative imaging assessment of cerebral edema facilitates genetic evaluation of early brain injury after} aneurysmal subarachnoid hemorrhage

Jane Yuan, BS ${ }^{1,2}$, Zach Zlepper ${ }^{1}$, Keshav Jayaraman, BS ${ }^{2,3}$, Atul Kumar, $M D^{1}$, Yasheng Chen, $P h D^{1}$, Joshua Osbun, $M D^{2}$, Umeshkumar Athiraman, $M D, M B B S^{3}$, Gregory Zipfel, $M D^{2}$, and Raj Dhar, MD, FRCPC ${ }^{1}$

Objective To validate automated assessment of sulcal CSF volume as a quantitative biomarker of GCE that can facilitate large-scale genetic studies of EBI. Background Despite decades of research targeting vasospasm and delayed cerebral ischemia, morbidity and mortality remain unacceptably high for aneurysmal subarachnoid hemorrhage patients. Early brain injury (EBI), a complex cascade originating from aneurysmal rupture and resulting in global cerebral edema (GCE), is now recognized to be a major determinant of poor outcome. Lack of a suitable biomarker that measures EBI in humans has hindered study of the molecular pathways most critical to its development. Effacement of cerebral sulci is used to qualitatively diagnose GCE. Recently, a semi-quantitative measure (Subarachnoid hemorrhage Early Brain Edema Score; SEBES, 0-4) has been demonstrated to capture severity of GCE, but relies on subjective, time-consuming, human assessment of brain imaging. Design/Methods We evaluated CT scans of aSAH patients for degree of GCE using: (1) manual SEBES; (2) automated volumetric measurement of CSF using a deep learning algorithm. Clinical GCE was defined as neurologic deterioration within 72 hours of $\mathrm{SAH}$, associated with sulcal effacement on imaging. Blood or saliva samples are being collected for subsequent genetic analysis. To validate that reduced sulcal CSF volumes can serve as a quantitative biomarker of GCE, we analyzed their association with SEBES scoring. Results Three hundred twenty aSAH patients were admitted between 2014 and 2018 (mean age 57 years, 73\% female, 34\% WFNS grade IV-V). Sixty-four (20\%) developed clinical GCE. Automated sulcal volumes were negatively correlated with SEBES scores in a preliminary cohort of 85 subjects (rho = $\left.-0.66, p=3 \times 10^{-11}\right)$. Mean sulcal volume was $23-\mathrm{mL}$ with high SEBES $(2-4)$ vs $66-\mathrm{mL}$ with low SEBES $\left(p=1.6 \times 10^{-8}\right)$. However, neither baseline sulcal volumes nor SEBES were associated with risk of symptomatic GCE. Conclusions Sulcal CSF volume may serve as an objective quantitative biomarker of GCE after SAH. However, admission measurements alone may not reflect the natural history of EBI. We now plan on applying this automated approach to study the evolution of edema over the first 72 hours in a larger cohort of aSAH patients with the goal of utilizing this for genetic studies of EBI.

Contact Email: dharr@wustl.edu

\section{Genome-wide analysis study in extracranial- and intracranial atherosclerosis in ischemic stroke patients using UK biobank}

Hye-Yeon Choi, $\mathrm{MD}, \mathrm{PhD}, \mathrm{MS}^{1}$, Scott Kulm, BEng ${ }^{2}$, and Olivier Elemento, $\mathrm{PhD}^{2}$

Objective This study aimed to identify genetic differences between extracranial- and intracranial atherosclerosis (ECAS and ICAS) using UK Biobank database. Background Atherosclerotic lesion distribution in cervicocephalic arteries varies with ethnicity. ECAS and ICAS have been suggested to have different pathogeneses. Intracranial atherosclerosis is more prevalent in Asian patients, whereas extracranial atherosclerosis is more common in individuals from western countries. The reasons for this discrepancy remain unknown. Design/Methods We carried out a preliminary genome-wide association study of 1,385 subjects with ICAS and 100 with ECAS from UK Biobank. Subject selection was performed using ICD 10 diagnosis code. Results The Manhattan plots showed that one single nucleotide polymorphism (SNP) could be considered as genome-wide significant, although not by a great amount. The SNP (rs75935776) appears to be related to ICAS, although the SNP is more relating to a difference between ECAS and ICAS rather than an ICAS alone risk factor. Conclusions Our preliminary results showed possible SNP related to ICAS. To understand differences between ICAS and ECAS, further welldesigned studies on various risk and genetic factors should be performed in patients with cerebral atherosclerosis.

Contact Email: hyechoi@gmail.com

\section{Coding region copy number variants in intracerebral hemorrhage}

Savvina Prapiadou, BSc ${ }^{1}$, Caspar Grond-Ginsbach, $P h D^{2}$, Padmini Sekar, $M S^{3}$, Jonathan Rosand, $\mathrm{MD}^{4}$, Iscia Lopes-Cendes, $\mathrm{MD}, \mathrm{PhD}^{5}$, Carl Langefeld, $\mathrm{PhD}^{6}$, and Daniel Woo, $\mathrm{MD}^{7}$

Objective To explore the association between rare copy number variants (CNVs) and the pathogenicity of intracerebral hemorrhage $(\mathrm{ICH})$. Background Recent studies have increasingly recognized the contribution of structural variants such as CNVs in the etiology of stroke. We sought to evaluate this approach for ICH. Design/Methods CNVs were identified (PennCNV software) from Affymetrix Human SNP Array 6.0 in 1,445 individuals from: Genetics of Anticoagulation and Hemorrhage (GOCHA), Genetic and Environmental Risk 
Factors for Hemorrhagic Stroke (GERFHS), the University of Joinville, Brazil and the University of Pisa, Italy. After quality control, 1,235 individuals ( $85.7 \%$ of the cohort) were analyzed: 472 controls (mean age $=67.2$ ) and 763 patients (mean age $=$ 68.2). CNVs were validated and mapped using the program noise-free-CNV. CNVs consisting of less than 20 SNPs, with minor allele frequency greater than $5 \%$ in $\mathrm{CNV}$ reference databases, in non-coding or regions with unclear mapping were excluded. Gene groups associated with relevant biological processes were analyzed by Gene Ontology Term Mapper. Results At least one rare, genic CNV was observed in $36.8 \%$ of the samples, with $\mathrm{ICH}$ patients having greater percent than controls $(39.7 \%$ vs $32.2 \% ; p=0.006$; OR $=1.42 ; 95 \% \mathrm{CI}=$ $1.10-1.82)$. However, the percent of CNVs that overlapped coding regions of 5 or more genes was higher in controls than cases $(6.1 \%$ vs $3.7 \%, p=0.04)$. Cases relative to controls were enriched for CNVs in the cholesterol biosynthesis pathway ( $5.9 \%$ vs $3.0 \%, p=0.0187)$. Neither blood vessel development genes $(7.7 \%$ vs $5.5 \%, p=0.13)$ nor ohnologs $(19.0 \%$ vs $17.2 \%$, $p=0.42)$ exhibited CNV enrichment in ICH cases. Interestingly, mosaic loss of the $\mathrm{Y}$ chromosome was associated with ICH development after adjusting for age and hypertension via logistic regression $(p=0.0036$; $\mathrm{OR}=6.17 ; 95 \% \mathrm{CI}=$ 1.81-20.95). Conclusions Genetic imbalance was more common in ICH cases than in controls which is consistent with ischemic stroke. The imbalance appears greater in cholesterolrelated genes consistent with prior epidemiology and genetic associations. Further analysis on the effect of genetic imbalance with outcomes is needed.

Contact Email: sprapiadou@gmail.com

\section{Early reduction in CSF volume captures the spectrum of cerebral edema after ischemic stroke}

Rajat Dhar, MD, FRCPC ${ }^{1}$, Yasheng Chen, $P h D^{1}$, Ali Hamzehloo, $M D^{1}$, Atul Kumar, $M D^{1}$, Laura Heitsch, $M D^{2}$, June $H e, M D$, $M S^{3}$, Ling Chen, $M D, P h D, M S^{3}$, Agnieszka Slowik, $M D, P h D^{4}$, Daniel Strbian, $M D, P h D^{5}$, and Jin-Moo Lee, $M D, P h D^{1}$

Objective To evaluate the reduction in CSF volume in the first 24-hours after acute ischemic stroke (AIS) as a quantitative imaging biomarker that captures the full spectrum of cerebral edema. Background Cerebral edema develops in the majority of patients after hemispheric stroke but only leads to mass effect and midline shift (MLS) in a minority. Even though deterioration usually occurs after $24-48$ hours, edema formation, the result of a complex biologic cascade in response to ischemic brain injury, begins in the first hours after AIS. Current measures of edema (neurologic deterioration, MLS) only represent the most severe and terminal stages of a process with a spectrum of severities. A quantitative biomarker of edema that captures the full spectrum would facilitate evaluation of the genetic basis for variation in edema severity and unlock investigations of mechanisms critical to edema formation. Design/Methods We selected subjects enrolled in the prospective GENISIS (Genetics of Early Neurologic InStability after Ischemic Stroke) study who presented within six hours of AIS and had both baseline and follow-up computed tomography (CT) imaging available. We applied a neural network algorithm to quantify CSF volume at both time points and calculated reduction in CSF volume $(\triangle \mathrm{CSF})$ as a potential biomarker of edema. We evaluated the relationship of $\triangle \mathrm{CSF}$ to neurologic deterioration ( $\triangle$ NIHSS), MLS, and functional outcome (mRS at 90-days). Results We had paired baseline and 24-hour imaging for 598 GENISIS participants from three sites (St. Louis, Krakow, Helsinki). The median $\triangle \mathrm{CSF}$ was $19-\mathrm{mL}$ (IQR 6-35), representing a $12 \%$ reduction from baseline. $\triangle \mathrm{CSF}$ exhibited a normal distribution with significant variation amongst patients within strata of NIHSS. $\Delta$ CSF was correlated with MLS $(r=0.51), \triangle$ NIHSS ( 1.3 point reduction per $10 \% \triangle \mathrm{CSF}$ ) and worse functional outcome (OR $1.29,95 \%$ CI 1.03-1.64). Conclusions $\triangle \mathrm{CSF}$ within 24 hours captures the variability in edema formation early after AIS and is meaningfully related to neurologic deterioration, midline shift, and outcome. We are now applying $\triangle \mathrm{CSF}$ as an endophenotype of edema for genetic studies.

Contact Email: dharr@wustl.edu

\section{Metabolomic biomarker predictors of vascular ischemia and infarct size in acute ischemic stroke: Initial results of Metabolome in Ischemic Stroke Study (MISS)}

Dharambir K. Sanghera, PhD, FAHA ${ }^{1,2,3,4}$, Chao Xu, $P h D^{6}$, Bahar M. Beaver, $M D^{5}$, Cynthia Bejar, $M S^{1,4}$, Bappaditya Ray, $M D, M B B S^{3,5}$, and Evgeny Sidorov, $M D, P h D^{3,5}$

Objective Purpose of this investigation was to identify circulating metabolites discriminating acute and chronic phase of ischemic stroke. Background Acute ischemic stroke (AIS) is the fifth leading cause of death in the United States. There is no reliable blood biomarker currently existing that can categorize and predict ischemic stroke. Metabolome profiling is a useful method to identify such biomarkers. Furthermore, the identification and characterization of these biomarkers to objective imaging data such as infarct volume can better delineate stroke pathophysiology and prognosis. Design/ Methods We have generated a global metabolomic profiles using serum samples of 62 AIS patients (124 in duplicate) at acute (72 hours) and chronic (3-9 months) phases and 8 healthy controls (16 in duplicate) using UPLC-MS/MS (Triple-TOF, ESI +ve and -ve; $\sim 10,000$ spectra. We also measured the volume of AIS using MR segmentation software. Results Our study detected several unknown metabolites to be significantly associated with AIS. We also detected 
20 known metabolites including oleyl ethanomide, glutamate, phosphocholine N-acetyleneuraminate, 1-palmtoloeylglycerol 16:1 whose concentrations were significantly perturbed during the acute phase compared to the chronic phase and some of these were associated with stroke volume (bonferroni corrected $p$ ranging from $3.5 \times 10^{-3}$ to 3.4 $\left.\times 10^{-8}\right)$. Several crucial pathways associated with metabolism of histidine, glycine-serine-threonine, alanine-aspartateglutamate, and nitrogen were overrepresented in patients with a set of metabolites during the acute phase when compared with healthy controls; whereas only glycine-serinethreonine pathway was overrepresented when compared between acute and chronic phase. Conclusions Our prospective study has identified circulating metabolites whose concentrations were altered at acute state of stroke. Some of these circulating molecules correlated significantly with the infarct volume with large-size strokes. Future confirmation in larger datasets would be needed to identify putative pathways underlie vascular ischemia which can be targeted for potential therapeutics and biomarker discovery.

Contact Email: Dharambir-sanghera@ouhsc.edu

\section{Volume of hemorrhagic}

transformation after acute ischemic stroke predicts neurological deterioration better than radiologic classification

A. Hamzehloo, $M D^{1}$, A. Kumar, $M D, P h D^{1}$, L.E. Heitsch, $M D^{1}$, D. Strbian, $\mathrm{MD}, \mathrm{FESO}^{2}$, T. Tatlisumak, $\mathrm{MD}, \mathrm{PhD}^{3}$, A. Stowik, $M D^{4}$, J.M. Lee, $M D, P h D^{1}$, and R. Dhar, $M D, F R C P C^{1}$

Objective We sought to evaluate whether the volume of hemorrhage better reflects neurologic outcomes than the radiologic classification of hemorrhagic transformation. Background Hemorrhagic transformation (HT) may contribute to neurologic deterioration after acute ischemic stroke (AIS). The current radiologic classification of HT distinguishes hemorrhagic infarction (HI) from a parenchymal hematoma $(\mathrm{PH})$. It is unclear whether this subjective qualitative grading is optimal for predicting the impact of HT on the outcome. Design/Methods We evaluated AIS patients with follow-up CT imaging from a prospective stroke genetics study. HT seen within 36 hours of AIS was classified using ECASS criteria. In addition, we outlined all confluent areas of hemorrhage to derive hemorrhage volume (HV). We calculated $\triangle$ NIHSS as the difference between baseline and 24-hour NIHSS. Early neurological deterioration (END) was defined as $\triangle$ NIHSS of -4 points or more. Association of HT grade and $\mathrm{HV}$ with $\triangle$ NIHSS and END were analyzed using linear regression and receiver-operating-curve testing. Results We analyzed 948 stroke patients with median NIHSS 7 (4-14), $64 \%$ receiving $\mathrm{tPA}$ and $\triangle \mathrm{NIHSS}+2(0-5)$. Two hundred ninety-four (31\%) had HT (146 HI1, 63 HI2, 42 PH1, and 43 $\mathrm{PH} 2)$. HT was associated with higher baseline NIHSS but not with tPA treatment or $\triangle$ NIHSS. END occurred in $113(12 \%)$ including 46 with HT (16\%) vs 67 (10\%) without HT ( $p=$ 0.02 ). However, amongst those with $\mathrm{HT}$, the radiologic grade was not associated with $\triangle$ NIHSS or END (20\% of PH2, $20 \%$ of $\mathrm{PH} 1$ vs $15 \%$ of $\mathrm{HI} 1 / \mathrm{HI} 2, p=0.40)$. In contrast, greater HV was associated with $\triangle$ NIHSS (adjusting for baseline NIHSS and $\mathrm{tPA}$, estimate -1.5 point per $10-\mathrm{mL}, p=0.0001$ ) and with END (those with END had median HV 7 vs 3-mL, $p=0.001$ ). Conclusions We demonstrated that ECASS classification did not accurately reflect the neurologic outcome after AIS and may not represent the most clinically meaningful measure of HT. Hemorrhage volume may provide a better quantitative biomarker to facilitate genetic studies of HT.

Contact Email: hamzhelooa@wustl.edu

\section{Genetic influences of stroke in African American hypertensives from GenHAT}

N.M. Davis Armstrong, $P h D^{1}$, V. Srinivasasainagendra, $M S^{1}$, H.K. Tiwari, $P h D^{1}$, D.K. Arnett, $P h D^{2}$, and M.R. Irvin, $P h D^{1}$

Objective To identify genetic variants associated with stroke in hypertensive African Americans (AAs) from the Genetics of Hypertension Associated Treatments (GenHAT) study. Background Hypertension (HTN) is a leading risk factor contributing to stroke. AAs have an earlier onset and more severe form of HTN and stroke, both attributed to social, environmental, and genetic factors. Identifying variants associated with stroke in individuals taking anti-hypertensive medication is critical to understanding genetic underpinnings to treatment response, as well as detecting at-risk individuals. Design/Methods We examined AA from GenHAT, an ancillary pharmacogenetic study to the Antihypertensive and Lipid Lowering treatment to prevent Heart Attack Trial (ALLHAT). ALLHAT participants were followed up to 8 years (1994-2002) for cardiovascular disease outcomes. Using 367 stroke cases and 6,530 controls randomized to chlorthalidone/CHL (thiazide diuretic, 192 cases) or lisinopril/LIS (ACE inhibitor, 175 cases), we performed a GWAS after imputing to the TOPMed Freeze 5 reference panel. Logistic regression models of 17,932,169 variants adjusting for age, sex, and ancestry (top 10 principal components) were used. Results Ten variants exceeded genomewide significance $(p=5.00 \mathrm{e}-08)$ in drug stratified analyses of stroke ( 1 for LIS, 9 for CHL). In the LIS stratum, rs 80001540 $(p=3.00 \mathrm{e}-08)$ was identified as a potential biomarker and is located downstream of PURPL. Three variants located in the GAB1 gene region ( $r 28925891 p=1.97 \mathrm{e}-07$; $r 114626322 p$ $=2.24 \mathrm{e}-07$; rs $115880531 p=2.33 \mathrm{e}-07)$ and one in SMAD9 (rs116429681 $p=1.27 \mathrm{e}-07)$ were nominally associated ( $p<$ $2.50 \mathrm{e}-07)$ with stroke. Nine variants were identified in the 
CHL stratum, including two variants in KANK3 (rs7258579 $p=3.56 \mathrm{e}-08$; rs $115048583 p=4.03 \mathrm{e} 08)$ and a missense variant in HTR3C (rs114941277 $p=5.31 \mathrm{e}-09)$. Conclusions In conclusion, we have identified 10 statistically significant variants associated with incident stroke in hypertensive AAs. Variants in KANK3, GAB1, and SMAD9 are of primary interest due to their roles in vascular development, angiogenesis, and HTN, respectively. Replication is warranted to determine whether these variants can be targeted for intervention in hypertensives.

Contact Email: nmda@uab.edu

\section{Sex and genetic susceptibility synergistically influence risk of stroke and myocardial infarction in middle- aged persons without risk factors}

G.J. Falcone, $M D, S c D, M P H^{1}$, J.N. Acosta, $M D^{1}$, C. Both, $B S^{1}$, A.C. Leasure BS ${ }^{1}$, K.N. Vanent, $B S^{1}$, R.B. Noche, $B S^{1}$, E. Kirsch $B S^{1}$, N. Petersen, $M D, M S c^{1}$, J. Schindler $M D^{1}$, L. Sansing, $M D^{1}$, T.M. Gill, $M D^{1}$, C.C. Matouk, $M D^{2}$, M. Gunel, $M D^{2}$, and K.N. Sheth, $M D^{1}$

Objective To test the hypothesis that sex and genetic susceptibility synergistically increase the risk of acute vascular events (AVE) in middle-aged adults without risk factors. Background Driven by aging-related physiological changes, the incidence of stroke and myocardial infarction rises rapidly in people aged $>40$ years. A significant proportion of AVE take place in people without vascular risk factors. Design/Methods We analyzed data from the UK Biobank, a prospective longitudinal study that enrolled persons aged 40 to 69 years. Our analysis was restricted to middle-aged participants, defined as those aged 40 to 60 years. Prevalent and incident cases of stroke (ischemic and hemorrhagic) and myocardial infarction were included. To quantify the genetic susceptibility to sustain an AVE, we constructed a polygenic risk score using 68 independent $\left(R^{2}<0.1\right)$ genetic variants known to associate $\left(p<5 \times 10^{-8}\right)$ with AVE. Participants were classified as having low, intermediate or high genetic risk according to tertiles of the score. We used Cox models for association and interaction testing. Results Of the 502,536 study participants enrolled in the UK Biobank, 303,295 (60\%) did not have any vascular risk factors. During the follow-up period, there were 5,746 AVEs, including 1,954 strokes and 3,792 myocardial infarctions. The cumulative risk of AVE was $0.12 \%(n=352)$, $0.46 \%(n=1,386)$ and $1.32 \%(n=4,008)$ at ages 40,50 and 60 years (test-for-trend $p<0.001$ ). The risk of AVE was 3 times greater in men than women (HR 3.30, 95\% CI 3.08-3.53). Compared to persons with low genetic risk, those with intermediate and high genetic risk had a 22\% (HR 1.22, 95\% CI $1.13-1.32$ ) and $52 \%$ (HR 1.52, 95\% CI 1.41-1.65) increase in risk of AVE, respectively. There was significant synergy (interaction) between sex and genetic susceptibility: compared to females with low genetic risk, males with high genetic risk had 4 times (HR 3.91, 95\% CI 3.58-4.26) the risk of AVE (interaction analysis $p<0.001$ ). Conclusions Genetic information constitutes a useful tool to risk stratify middle age adults without vascular risk factors. The synergistic effect of sex and genetic susceptibility points to specific subgroups that could benefit from aggressive preventive interventions (figure 1).

Contact Email: guido.falcone@yale.edu

\section{Effects of the BDNF Val66Met polymorphism on acute stage measures of function in young stroke patients}

R.G. Braun, $M D, P h D^{1}$, S.J. Kittner, $M D, M P H^{1}$, K.A. Ryan, $M P H^{2}$, and J.W. Cole, $M D, M S^{1}$

Objective To assess the effects of the BDNF Val66Met polymorphism in a cadre of young stroke patients aged 15-49 years. Background Aging effects on CNS recovery after stroke are known to interact with the effects of genetic polymorphisms, including those affecting the BDNF gene. Multiple prior studies have examined the effect of the BDNF Val66Met polymorphism in older stroke patients, but the effect of this polymorphism in younger stoke patients remains incompletely characterized. To address this knowledge gap, we present findings from a study of first-ever ischemic stroke among patients aged 15-49 years. Design/Methods Eight hundred twenty-nine patients with a first ischemic stroke (Average age $=41.4$ years, $\mathrm{SD}=6.9$ ) were recruited from the Baltimore-Washington region. Genotyping was performed at the Johns Hopkins University Center for Inherited Disease Research (CIDR). Data cleaning and harmonization were done at the GEI-funded GENEVA Coordinating Center at the University of Washington. Our sample contained 165 Met carriers and 664 non-Met carriers. Modified Rankin scores were obtained at the time of discharge from the acute hospital and binarized for analysis into "Poor" vs "Good" outcomes (mRS 0-2 vs 3+). Results Our results demonstrate that the Val66Met polymorphism exerts a negative effect on mRS scores in this cadre of younger stroke patients. Our model, which accounted for Sex, Age, and principal components, showed a significant protective effect of the Val allele (adjusted OR 0.68, 95\% CI 0.468-0.997 $p=0.05$ ). Conversely, Met carriers were at proportionally greater risk for poor mRS outcomes (adjusted OR 1.46, 95\% CI 1.003-2.137, $p=0.05$ ). Conclusions Negative effects on stroke functional outcomes in younger carriers of the BDNF Val66Met polymorphism have previously been posited to arise as an effect of advancing age under the tenets of the resource modulation hypothesis. This perspective is challenged by the presence of negative Val66Met effects on functional outcomes in our cadre of young stroke survivors. Further longitudinal studies are 


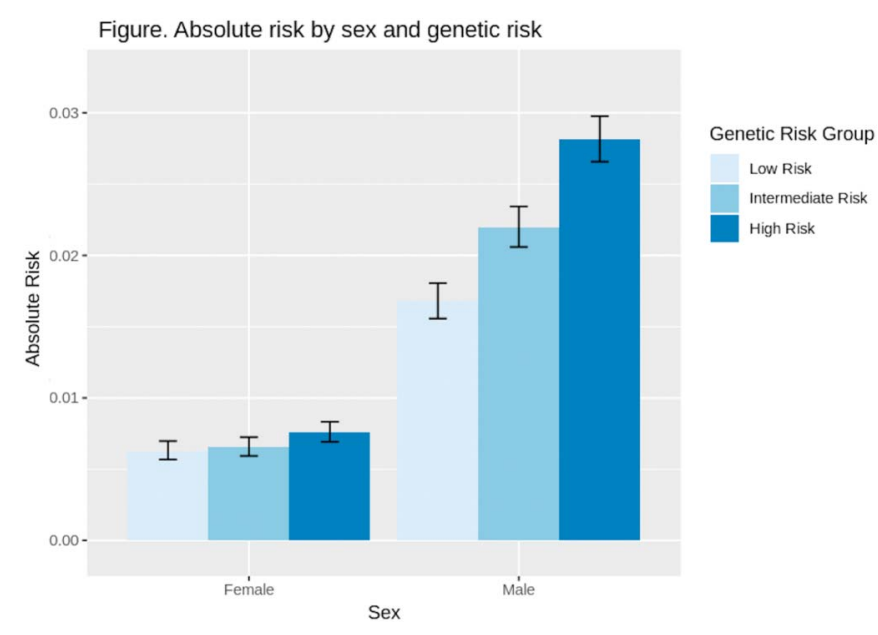

needed to determine whether the effects of this polymorphism extend to later post-acute recovery time points and will be a focus of future research.

Contact Email: Robynne.Braun@umm.edu

\section{Epigenome-wide meta-analysis of cerebral white matter hyperintensities on MRI and integrated omics analysis}

Yunju Yang, $M P H^{1}$, Hieab Adams, $P h D^{2}$, Joshua C. Bis, $P h D^{3}$, Shuo Li, $P h D^{4}$, Min A. Jhun, $P h D^{5}$, Michelle Luciano, $P h D^{6}$, Alexander Teumer, $P h D^{7}$, Sharon L.R. Kardia, $P h D^{5}$, Ian Deary, $P h D^{6}$, Hans J. Grabe, $M D^{7}$, Lenore J. Launer, $P h D^{8}$, W.T. Longstreth, $M D^{3}$, M. Arfan Ikram, $M D^{2}$, Sudha Seshadri, $M D^{9}$, and Myriam Fornage, $P h D^{1}$

Objective To identify novel DNA methylation (DNAm) loci influencing the burden of cerebral white matter hyperintensities (WMH) on MRI. Background Cerebral WMH are an indicator of cerebral small vessel disease, a major risk factor for vascular dementia and stroke. DNAm may contribute to the molecular underpinnings of WMH, which are highly heritable.

Design/Methods We included 6,019 middle-aged to elderly individuals from 11 population-based cohorts, who were free of dementia and stroke and were of African (AA) or European (EA) descent. In each cohort, association between WMH volume and each $\mathrm{CpG}$ was tested within ancestry using a linear mixed model, adjusted for age, sex, total intracranial volume, white blood cell count, technical covariates, smoking, body mass index and blood pressures. Meta-analyses were performed within ancestry and in the total sample. We identified DMRs using region-based $p$-values accounting for spatial correlations among CpGs. Finally, we performed colocalization analyses of methylation quantitative trait loci (mQTLs) and WMHassociated genetic loci using a Bayesian method. Results No individual $\mathrm{CpG}$ reached epigenome-wide significance, but suggestive novel associations were identified with cg17577122 (CLDN5, $p=2.39 \mathrm{E}-7$ ), cg24202936 (LOC441601, $p=3.78 \mathrm{E}-$ 7), cg03116124 (TRIM67, $p=6.55 \mathrm{E}-7$ ), and cg04245766 (BMP4, $p=3.78 \mathrm{E}-7$ ). Gene set enrichment analyses implicated pathways involved in regulation of cell development and differentiation, especially of endothelial cells. We identified 11 DMRs (Sidak-adjusted $p<0.05$ ) including two mapping to blood pressure-related genes (HIVEP3, TCEA2). Genes mapping to DMRs were enriched in biological processes related to metabolism and transport of lipoprotein. Finally, the colocalization analysis provided evidence that 4 GWAS loci $(2 \mathrm{p} 16,7 \mathrm{q} 32$, 14q32, and 6q25) colocalized with mQTLs. Conclusions We identified novel epigenetic loci associated with $\mathrm{WMH}$, which may provide new clues about pathogenesis. We also show that DNAm plays a major role at known WMH loci.

Contact Email: myriam.fornage@uth.tmc.edu

\section{Somatic activating mutations in cerebral aneurysms}

Hirofumi Nakatomi, $M D, P h D^{1}$

Objective We performed phenotype-genotype analysis in the intracranial aneurysms to clarify the role of somatic genetic alterations within the aneurysm wall. Background Intracranial aneurysms can cause devastating stroke, resulting in high morbidity and mortality. The pathogenesis of cerebral aneurysm formation and rupture is complex, involving environmental and genetic factors. The role of somatic genetic alterations within the aneurysm wall is unknown. Design/ Methods We analyzed 34 sporadic cerebral aneurysm paired samples from patients by means of exome sequencing. Using exome sequencing we tested for the presence of a somatic mutation in 34 individuals. We have identified 36 mutations in 
fusiform aneurysms, 154 mutations in saccular aneurysms and 4 among these mutations were shared both in fusiform and saccular cerebral aneurysms. In fusiform aneurysms, PDGFRB activating mutations was robust. The functional effects of these mutations on downstream signaling were investigated, using phosphorylation specific antibodies and cultured cells expressing PDGFRB variants. Results Here, we report 4 kinds of PDGFRB somatic activating mutation in fusiform aneurysm patients and two KY somatic activating mutations in saccular aneurysm patients. We detected somatic activating PDGFRB mutations in the juxtamembrane domain and kinase activation loop in 4/13 fusiform aneurysms (0/21 saccular aneurysms, Fisher's Exact Test $p<0.001)$. Tissues from patients with fusiform aneurysms harbored admixtures of mutant alleles that ranged from $5 \%$ to approximately $40 \%$. PDGFRB mutant, but not wild type in cultured cells were found to have overactive auto-phosphorylation with downstream activation of ERK. Expression of discovered variants demonstrated non-ligand dependent auto-phosphorylation. Conclusions Somatic gainof-function mutations in PDGFRB are unique mechanism in the pathophysiology of fusiform cerebral aneurysms and suggest a potential role for targeted therapy with kinase inhibitors.

Contact Email: hirofuminakatomi@gmail.com

\section{GWAS of SiGN with TOPMed imputation reference panel uncovers novel stroke loci}

Brady J. Gaynor, $M S^{1}$, Kathleen Ryan, $M P H^{1}$, Patrick F. McArdle, PhD ${ }^{1}$, Thomas J. Jaworek, $B S^{1}$, John W. Cole, MD, $M S^{1}$, Steven J. Kittner, $M D^{1}$, Huichun $\mathrm{Xu}, \mathrm{MD}, P h D^{1}$, and Braxton D. Mitchell, $\mathrm{PhD}^{1}$; for the SiGN Network

Objective To perform an updated genome-wide association analysis of the SiGN Network using the TOPMED imputation reference panel. Background Genotype imputation, the process of leveraging haplotypes observed in a reference panel to infer unobserved genotypes in other samples, is widely used in genetic studies. The power of imputation is a function of the size and diversity of the reference panel, with larger and more diverse panels tagging more variants and including more lower frequency variants. We have previously performed a genome-wide (GWA) analysis of the SiGN study that was based on the 1000G and Genome of the Netherlands (GoN) imputation reference panel ( $\mathrm{n}=$ 1,591 samples). For this study we performed a re-analysis of SiGN after re-imputation using the TOPMed reference panel ( $\mathrm{n}>62,000$ samples) to determine if novel associations could be identified. Design/Methods We performed a meta-analysis of 12,577 stroke cases and 25,643 controls, first using the $1000 \mathrm{G} / \mathrm{GoN}$ imputation reference panel and then again using the TOPMed reference panel. These analyses were restricted to subjects of European ancestry. Results As expected, the TopMed-imputed GWAS re- identified the 5 stroke loci that were reported in the original GWAS analysis based on the $1000 \mathrm{G} / \mathrm{GoN}$ imputation. Additionally, the TopMed-imputed GWAS identified 6 novel loci associated with overall stroke or one of its subtypes, including 4 loci for which the minor allele frequency corresponding to the peak SNP ranged from $1 \%$ to $5 \%$. Among these loci is rs62066360 (Odds Ratio $=1.28, p$-value $=3.66 \mathrm{E}-08)$, which is located in the gene MYBBP1A and within enhancers for multiple brain tissues and adipose tissue besides being an expression quantitative trait locus in skeletal-muscle tissue. The peak SNPs at all 6 of these novel loci also showed evidence for association in MEGASTROKE ( $\mathrm{n}=67,142$ cases), with $p$-values ranging from 0.03 to $5.76 \mathrm{E}-4$. Conclusions Using the larger and more diverse TOPMed imputation refence panel enabled identification of six novel stroke-associated loci that had not previously been identified using the smaller 1000 Genomes/ GoN reference panel.

Contact Email: bgaynor@som.umaryland.edu

\section{Novel transcripts miR-1301, miR- 130, and miR-629 influence early neurologic outcome}

Alexis N. Simpkins, $M D, P h D^{1,2}$, Kory Johnson, $P h D^{2}$, Zurab Nadareishvili, $M D, P h D^{3}$, Devon Kelley, $B S^{2}$, Richard Leigh, $M D^{2,4}$, Marie Luby, $P h D^{2}$, Richard Benson, $M D, P h D^{5}$, Amie $H$ sia, $M D^{6}$, John Lynch, DO, $M P H^{2}$, John Hallenbeck, $M D^{2}$, and Lawrence Latour, $\mathrm{PhD}^{2}$

Objective Identify time dependent miRNA-mRNA transcript interactions in acute ischemic stroke (AIS) patients influence by time and early changes in ischemic volume (ECS), an imaging biomarker of response to thrombolysis and early neurologic improvement (ENI). Background Micro RNA (miRNA) regulate gene transcription induced by cerebral ischemia. Currently, time dependency and the influence of thrombolysis on such miRNA expression and downstream effects on gene transcription and clinical outcome is unknown. Design/Methods Enrolled patients $(\mathrm{n}=22)$ had MRI and blood sample collection at baseline, 2-hours, and 24hours post baseline MRI. Blood samples from patients with a known last seen normal (LSN) up to 24 hours were analyzed, excluding post-thrombolysis samples to select time dependent miRNA. ECS (difference in ischemic volume before and 2-hour post-thrombolysis) were measured using automated co-registered MRI sequences and correlated with transcript profiles collected 2-hours post-thrombolysis. RNASeq was performed with HiSeq 25,000 Illumina platform. After correcting for age, gender, and race, leave one out testing was used to select transcripts that correlated with the ECS or time from LSN (FDR corrected $p$-value $<0.05$ ). IPA Ingenuity MicroRNA filter was used to identify miRNA-mRNA interactions. Results Transcripts miR-1301 and miR-629 
positively and miR-130 negatively correlated with LSN and had miRNA-mRNA interactions with 4 mRNA transcripts that significantly correlated with ECS (lactate dehydrogenase A, solute carrier family 44 member 1 , ubiquitin specific peptidase 33 , and dual specificity phosphatase 3 ). These mRNA negatively correlated with ECS, meaning patients with larger reductions in ischemic volume were less likely to have the following expected downstream effects from these miRNAmRNA interactions reported in the literature, including, hypoxia inducible factor- 1 alpha degradation, thrombus formation, abnormal glutamate clearance, blood brain barrier disruption, cerebral edema, and angiogenesis. Conclusions We report 3 novel miRNA selected by MRI biomarker of ENI that may have potential as unique therapeutic targets in AIS patients.

Contact Email: alexis.simpkins@neurology.ufl.edu

\section{Single-nuclei RNA-seq to ascertain human tissue}

Jorge L. Del-Aguila, $P h D^{1,2,3,4}$, Jin-Moo Lee, $M D, P h D^{5,6}$, Carlos Cruchaga, $P h D^{1,2,3,4}$, and Oscar Harari, $P h D^{1,2,3,4}$

Objective Bulk methods of RNA sequencing (RNA-seq) has shown to provide valuable insights for many biological process, diseases and traits, buy processing tissue homogenates that average out the differences among cell-types. Single-cell RNA-seq produces a detailed molecular atlas can reveal the subtle changes but requires that cell resuspension and library preparation to be done from fresh tissue. This requirement prohibits the study of highly informative frozen human tissue stored in biorepositories. Single-nuclei RNAseq (snuclRNA-seq) is an alternative to this methodology. This abstract summarizes our finding while employing snuclRNA-seq to analyze frozen brain tissue, plasma and clots. Background Alzheimer's Disease $(\mathrm{AD})$ is the most common form of dementia. This neurodegenerative disorder is associated with neuronal death and gliosis heavily impacting the cerebral cortex. $\mathrm{AD}$ has a substantial but heterogeneous genetic component, presenting both Mendelian and complex genetic architectures. Using bulk RNA-seq from parietal lobes and deconvolution methods, we previously reported that brains exhibiting different $\mathrm{AD}$ genetic architecture exhibit different cellular proportions. Here, we sought to directly investigate $\mathrm{AD}$ brain changes in cell proportion and gene expression using single cell resolution. Design/Methods We generated unsorted single-nuclei RNA-sequencing data from brain tissue. We leveraged tissue donated from a carrier of a Mendelian genetic mutation, PSEN1 p.A79V, and two family members who suffer from sporadic $\mathrm{AD}$, but do not carry any autosomal mutations. We evaluated alternative alignment approaches to maximize the titer of reads, genes and cells with high quality. In addition, we employed distinct clustering strategies to determine the best approach to identify cell clusters that reveal neuronal and glial cell types and avoid artifacts such as sample and batch effects. We propose an approach to cluster cells that reduces biases and enables further analyses. Results We identified distinct types of neurons, both excitatory and inhibitory, and glial cells, including astrocytes, oligodendrocytes, and microglia among others (figure 2). In particular, we identified a reduced proportion of excitatory neurons in the Mendelian mutation carrier, but a similar distribution of inhibitory neurons. Furthermore, we investigated whether single-nuclei RNA-seq from human brains recapitulates the expression profile of Disease Associated Microglia (DAM) discovered in mouse models. We also determined that when analyzing human single-nuclei data it is critical to control for biases introduced by donor specific expression profiles. Conclusions We propose a collection of best practices to generate a highly detailed molecular cell atlas of highly informative frozen tissue stored in brain banks. Importantly, we have developed a new web application to make this unique single-nuclei molecular atlas publicly available. Finally, we have generated singlenuclei RNA-seq from circulating blood and clots from four donors and capture transcriptomic differences between clots and circulating blood. Our preliminary results show that this technology can be employed to analyze thrombectomy clots.

Contact Email: harario@wustl.edu

\section{Genetic influences on early neurological instability after acute ischemic stroke: GENISIS results}

Laura Ibanez, $P h D^{1}$, Laura Heitch, $M D^{1}$, Caty Carrera, $M D^{2}$, Daniel Strbian, $\mathrm{MD}, \mathrm{PhD}^{3}$, Agnieszka Slowik, $\mathrm{MD}, \mathrm{PhD}^{4}$, Antonio Arauz, $\mathrm{MD}, P h D^{5}$, Israel Fernandez, $P h D^{2}$, Joan Montaner, $\mathrm{MD}, \mathrm{PhD}^{2}$, Jin-Moo Lee, $\mathrm{MD}, P h D^{1}$, and Carlos Cruchaga, $\mathrm{PhD}^{1}$

Objective To identify genetic loci related to early neurological changes after ischemic stroke using a genome wide association study (GWAS). Background Following acute ischemic stroke (AIS) onset, neurological deficits can be highly unstable within the first 24 hours. We hypothesized that this early change, defined here as $\Delta$ NIHSS24h (NIHSS baseline - NIHSS 24 hours), could serve as a quantitative phenotype to capture the genetic architecture of ischemic brain injury. Design/Methods AIS patients were prospectively enrolled between 2008 and 2019 at 7 sites (St Louis, Barcelona, Helsinki, Krakow, Korea, Costa Rica and Mexico). NIHSS scores were obtained within 6 hours and again at 24 hours after stroke onset. Genome-wide genotyping was generated for rare and common variants, imputing up to 6 million single nucleotide polymorphisms (SNPs) for all subjects $(\mathrm{N}=5,487) . \Delta \mathrm{NIHSS} 24 \mathrm{~h}$ was used as a quantitative trait 
Figure 2 (Abstract 15) Single-nuclei RNA-sequencing analyses demonstrating distinct types of neurons and glial cells within thrombectomy clots as compared to circulating blood

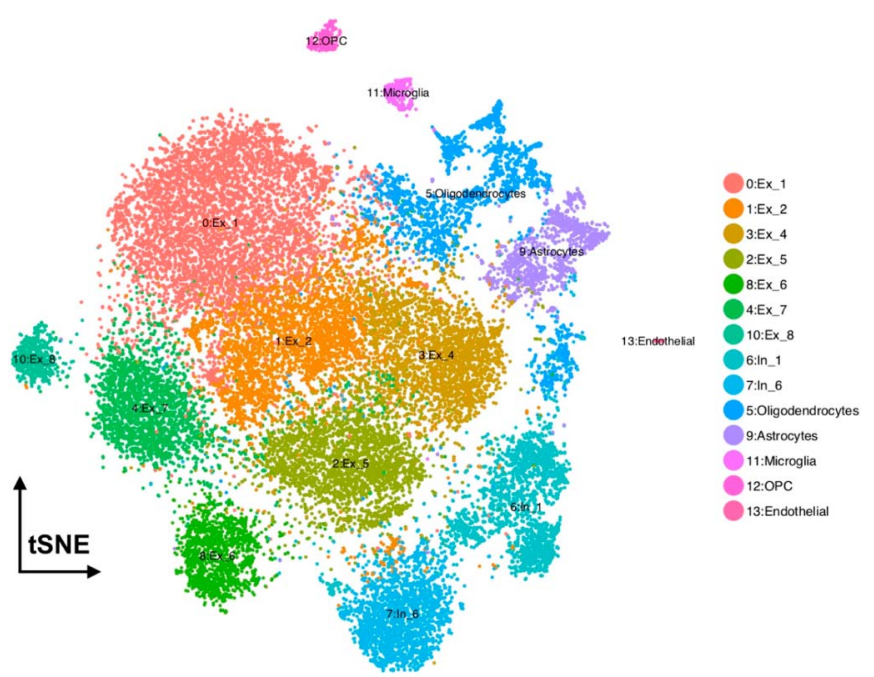

in an association model, with covariates that included baseline NIHSS, age, sex, PCA1, PCA2 and genotyping round. All samples were analyzed together using Plink 1.9. They are also analyzed by site and then, we used Mantra to perform a transethnic meta-analysis. Results We found one GWAS significant locus in the joint-analysis ( $r$ 16838295 $-p=2.84 \times$ $10^{-08}$ ) and two suggestive loci (rs114248865 and rs10807797) (figure 3). After deep functional annotation and Mendelian randomization analyses, the top association in chromosome 2 was driven by ADAM23. Conclusions Early neurological outcome after AIS is strongly influenced by genetics. ADAM 23 is a neuronal transmembrane protein involved in the bridging of pre- and post-synaptic membranes. It appears to play a role in modulating trans-synaptic excitability, suggesting a role in excitotoxicity in the setting of AIS.

Contact Email: ibanezl@wustl.edu

\section{Genome-wide association study of early-onset ischemic stroke identifies novel locus on chromosome 12 near BCL7A/MLXIP}

T. Jaworek, $B S^{1}$, and S.J. Kittner, $M D^{1}$; for the Early-Onset Ischemic Stroke Consortium

Objective Genetic studies of early-onset disease have been an effective strategy to identify novel pathways and drug targets relevant to later-onset disease. Background Few studies have investigated the role of common genetic variation in the etiology of early-onset ischemic stroke (IS). Design/Methods We performed a GWAS meta-analysis of 38 studies from 10 countries, comprised of 5,847 IS cases of European ancestry under age 60 and 32,533 controls. Results We identified two genome-wide significant $\left(p<5 \times 10^{-8}\right)$ loci (figure 4$)$. The

Figure 3 (Abstract 16) Manhattan plot showing GWAS results for early neurological changes after ischemic stroke in 5,487 case subjects

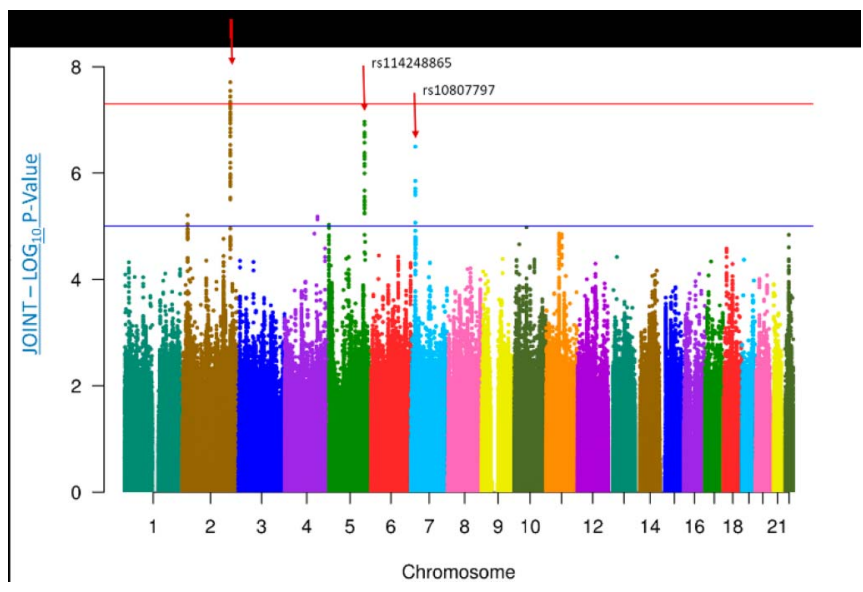


$\mathrm{ABO}$ locus has previously been associated with venous thrombosis and ischemic stroke in predominantly older adults, but the effect size of our top SNP (OR 1.18; $p=9.1 \times$ $10^{-12}$ ) is larger than the effect size for this same SNP in MEGASTROKE (OR: $1.05 ; p=6.5 \times 10^{-5}$ ). The lead SNP at the BCL7A/MLXIP locus is a novel GWAS finding for stroke (OR 1.14, 95\% CI 1.08-1.19; $p=1.7 \times 10^{-8}$ ) and is noteworthy because of prior reports linking SNPs in these genes to $\mathrm{BMI}$ and blood pressure. Conclusions We identified a novel locus that is near variants associated with BMI and blood pressure. Further studies are needed to confirm this locus, examine subtype specificity, and determine its function. The larger effect size observed at the $\mathrm{ABO}$ in this early-onset IS sample compared to older-onset IS samples is consistent with a larger role for prothrombotic mechanisms in early-onset IS.

Contact Email: skittner@som.umaryland.edu

\section{Online neurodegenerative trait integrative multi-omics explorer}

J.A. Bahena, $B E^{1,2}$, F.H.G. Farias, $P h D^{2}$, K.A. Mihindukulasuriva, $P h D^{2}$, J.P. Budde, $B A^{2}$, C. Cruchaga, $P h D^{2}$, and O. Harari, $P h D^{2}$

Objective To develop a new, online catalogue of Genome Wide Association Studies (GWAS) dedicated to quantitative neurological disorder endophenotypes. Additionally, to extend and incorporate previously described GWAS from our group to 59 cerebrospinal fluid (CSF) analytes. Background GWAS results are typically deposited in large databases such as the GWAS Catalog or $\mathrm{dbGaP}$. However, these repositories cover a vast array of phenotypes, complicating cross-comparisons specific to neurological disorders. Therefore, we compiled the Online Neurodegenerative Trait Integrative Multi-Omics Explorer (ONTIME, http://ngi.pub/ONTIME/) dedicated to quantitative neurodegenerative and complex disease phenotypes. Part of this neurology-based visualization tool includes an extended GWAS of 59 CSF analytes for two independent and expanded cohorts. Design/Methods ONTIME was built from the PheWeb code base (https://github.com/statgen/pheweb). Summary statistics were filtered and harmonized using dbSNP's Human Build
150 as reference. All genomic positions are on GRCh37. The extended GWAS comprised 672 subjects from the Alzheimer's Disease Neuroimaging Initiative (ADNI) cohort and the Washington University Knight Alzheimer's Disease Research Center (Knight-ADRC) cohort. We tested over 5.8 million SNPs for association to 59 analytes collected from CSF. We controlled for age, gender, the first two principle components. Results ONTIME contains summary statistics for more than 200 quantitative neurodegenerative endophenotypes. Users can query the association to variants by therapeutic category, phenotype, gene, or variant. This tool provides interactive visualization of summary statistics in the form of Manhattan plots, LocusZoom, and PheWAS plots for comparisons across all endophenotypes available in the explorer. Within this browser, we included summary statistics for the extended GWAS of 59 CSF analytes. We identified 7 analytes associated with 332 SNPs at a study-wide significance level of $p$ $<1.46 \times 10^{-10}$. While 5 of these analytes were identified in the original analysis, two (HCC4 and Trefoil Factor-3) had not previously reached study-wide significance. Conclusions ONTIME provides a unique opportunity to leverage GWAS of neurodegenerative disease endophenotypes to identify shared pathways for disease pathogenesis in complex diseases such as stroke.

Contact Email: Jorge.A.Bahena@wustl.edu

\section{Identification of cerebrospinal fluid (CSF) protein quantitative trait loci ( $\mathrm{pQTLs)}$ for stroke risk, recovery and other neurological disorders elucidates mechanisms underlying stroke}

Chengran Yang, $M S^{1,2,3,4}$, Fabiana Geraldo Farias, $P h D^{1,2,3,4}$, Kathie Mihindukulasuriya, $P \mathrm{D}^{1,2,3,4}$, Richard Davenport, $M S^{1,2,3,4}$, Fengxian Wang, $P h D^{1,2,3,4}$, John Budde, $B A^{1,2,3,4}$, Laura Ibanez, $P h D^{1,2,3,4}$, Herve Rhinn, $\mathrm{PhD}^{5}$, Carlos Cruchaga, $P h D^{1,2,3,4}$, and Oscar Harari, $P h D^{1,2,3,4}$

Figure 4 (Abstract 17) Manhattan plot showing GWAS results for early-onset ischemic stroke in 5,847 cases of European ancestry under age 60 and 32,533 controls

EUROPEAN Meta-Analysis - All Ischemic Stroke

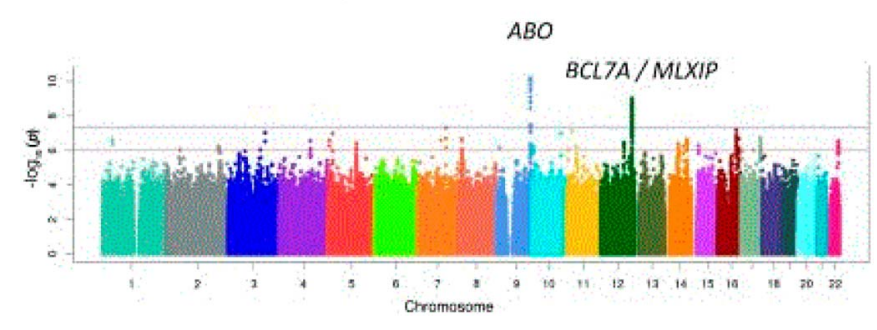


Objective We aim to identify the genetic architecture that govern protein levels and how are these proteins associated with stroke risk and additional neurodegenerative traits. Background Stroke ranks the second in diseases leading cause of death worldwide as of 2015. It has multiple etiologies and risk factors, but the underpinning mechanism remains largely unknown. Using genome-wide association studies (GWAS), researchers from large consortium have identified 32 stroke risk loci that can be used to build prediction models. Given successful GWAS requires large sample sizes, scientists have gradually focused on linking intermediate traits, such as protein level, with genomic variations, because these intermediate traits can elucidate the molecular mechanisms between the genomic variation to the disease. As a key intermediate trait, protein level has been widely used in the disease diagnosis. To infer the protein function in causal pathway of stroke statistically, we used two sample Mendelian Randomization (MR). MR estimates the causal extent of the exposure (protein) on the outcome (ischemic stroke risk). This strategy provides a mean to identify novel biomarkers. Design/Methods Here, we have profiled 1,305 proteins in 510 CSF, 337 plasma and 305 brains samples from a cohort of Alzheimer's disease cases and controls. Several known proteins associated with stroke risk are included such as von Wille brand factor, C-reactive protein, Fibrinogen, P-selectin, E-selectin and Vitronectin. We first performed protein GWAS to identify pQTLs from samples passing QC by employing linear regression models after adjusting for age, gender, two genetic principle components. We next performed MR using these pQTLs and stroke-risk and outcomes GWAS summary statistics. Results For CSF, we identified 82 proteins with cis-pQTLs study-wide significant $(p<7.01 \times$ $10^{-11}$ after Bonferroni correction), replicating 28 previously reported cis-pQTLs from CSF (e.g., rs2673908 associated with the protein Siglec-9, $\left.p=6.1 \times 10^{-160}\right)$ reported before. We also discovered 54 novel cis-pQTLs and 20 novel trans$\operatorname{pQTL}\left(p<7.01 \times 10^{-11}\right)$ for CSF protein levels. Using MR, we did not find a significant causal relationship between von Willebrand factor and Ischemic stroke $(p=0.94)$. pQTL and MR analyses for plasma and brain proteomics are ongoing. Conclusions This is the largest multi-tissue proteomic study performed so far. We have identified a large number of novel and known cis and trans pQTL loci. MR analyses in this data will allow us to identify novel biomarkers and proteins implicated on stroke risk, onset and outcome.

Contact Email: chengranyang@wustl.edu

\section{An inverse relationship between cerebral microbleeds and migraine burden in CADASIL}

E.D. Goldstein, $M D^{1,2}$, M.B. Badi, $M D^{1}$, J. Klaas $M D^{3}$, E. Lesser, $M S^{1}$, D.O. Hodge, $M S^{1}$, K.H. Wong, $B S^{2}$, K. Aitken, $M P H$, $C C R P^{2}$, H. Baradaran, $M D^{2}$, R.D. Brown Jr, $M D, M P H^{3}$,
D.W. Dodick, $M D^{4}$, T. Rozen, $M D^{1}$, D. Capobianco, $M D^{1}$, M.P. Lin, $M D, M P H^{1}$, J.F. Huang, $M D^{1}$, J.J. Majersik, $M D, M S^{2}$, and J.F. Meschia, $M D^{1}$

Objective To evaluate the relationship between cerebral microbleed (CMB) burden and migraine-related disability in patients with cerebral autosomal dominant arteriopathy with subcortical infarcts and leukoencephalopathy (CADASIL). Background CADASIL is caused by a mutation in the NOTCH3 gene leading to progressive central nervous system small vessel disease. Patients often have coexisting migraine with aura (MA), though its relationship to the underlying CADASIL pathophysiology remains unclear. Design/Methods We performed a prospective, crosssectional, observational study of a series of adults with CADASIL who had a brain MRI with a hemosiderinsensitive sequence. The Microbleed Anatomical Rating Scale (MARS) was used to quantify CMB burden in which ordinal advancing score signifies greater CMB quantity. The Migraine Disability Assessment Questionnaire (MIDAS) was used to quantify migraine related disability $(0-5$, $6-10,11-20,>20$ meaning no, minimal, moderately limited, and severely limited disability respectively). Brain MRIs were assessed blinded to clinical data or questionnaire results. Results Twenty-three CADASIL patients with and without MA were included (mean enrollment age, 59 years; $65 \%$ women). A total of $43.5 \%(10 / 23)$ had $\geq 1$ on MIDAS (mean 26, range 0-197) and 56.5\% (13/23) had $\geq 1$ CMB (MARS range: $0-53$ ). Those with $\geq 1 \mathrm{CMB}$ s were significantly less likely to have a MIDAS $\geq 1$ (odd ratio 0.05 ; 95\% CI 0.01-0.49; $p=0.01$ ). The inverse relationship remained significant after adjusting for age at MRI scanning and MIDAS interview. Conclusions A unique inverse relationship was noted between $\mathrm{CMB}$ burden and migrainerelated disability amongst CADASIL patients. Future investigations may serve to understand the relationship of MA phenotype on CMBs in CADASIL, the influence of the trigeminovascular system on cerebral small vessel disease and on novel therapeutic pathways to forestall small vessel disease progression in CADASIL.

Contact Email: eric.goldstein@hsc.utah.edu

\section{Prevalence of monogenic disorders in young stroke patients: Preliminary results from the GENE_YAS study of the CRCS-K}

Hong-Kyun Park, MD, MSc ${ }^{1}$,Keon-Joo Lee, MD, MSc ${ }^{2}$, Joon Seol $\mathrm{Bae}, \mathrm{PhD}^{3}$, and Hee-Joon Bae, $\mathrm{MD}, \mathrm{PhD}^{2}$; on behalf of CRCS-K Investigators

Objective To evaluate the prevalence of 14 monogenic disorders which are associated with stroke, and to develop the algorithms to screen monogenic disorders in young stroke 


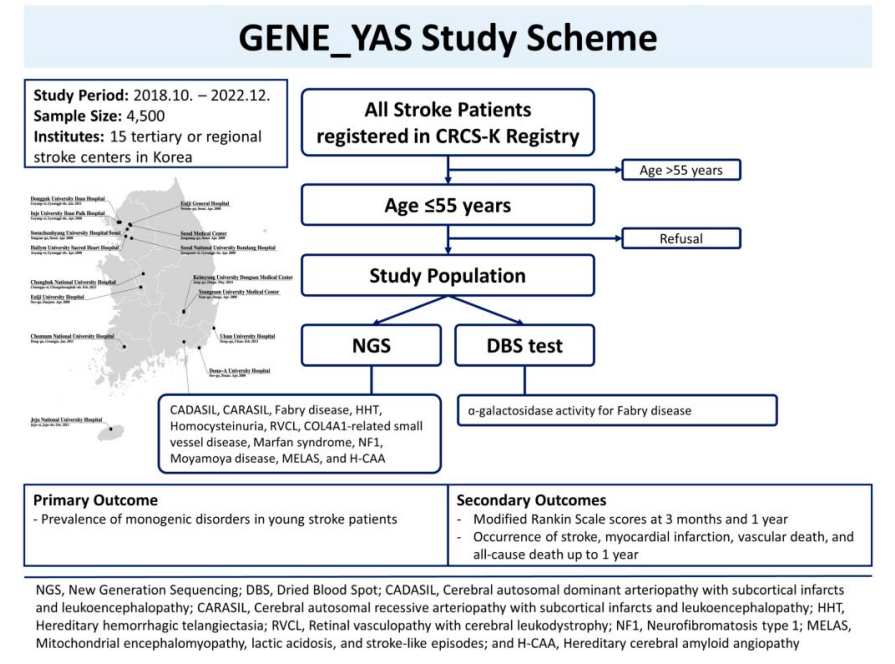

patients. To evaluate the prevalence of 14 monogenic disorders which are associated with stroke, and to develop the algorithms to screen monogenic disorders in young stroke patients. Background Although the identification of known monogenic disorders causing stroke might be important both for patients themselves and family members for knowing patient's prognosis and genetic counseling, there had been no large prospective study on their prevalence and screening flow for stroke population. Previously, we presented the concept and design of this study. Here we report the results of this study of the first 6 months. Design/Methods This ongoing study is based on a prospective nationwide multicenter stroke registry (Clinical Research Collaboration for Stroke in Korea, CRCS-K), all patients aged 55 years or younger would be consecutively enrolled unless they refuse to give consent. Patients are screened by a gene panel of 14 known stroke related monogenic disorders which uses the New Generation Sequencing method. The final sample size is planned to be 4,500 , and we were available to report this preliminary result. The figure 5 presents the study scheme. Results From October 2018 to March 2019, a total of 275 patients (46.4\%; age $44.8 \pm 8.5$ year) gave their consent to this study among 593 eligible patients and underwent the screening process. Thirty-five (12.7\%) patients had pathogenic or likely-pathogenic mutation identified by the gene panel. Mutation in ABCC6 gene was most frequently observed $(14,5.1 \%)$ followed by $\operatorname{RNF} 213$ (13, 4.7\%), NOTCH3 (4, 1.5\%), FBN1 (3, 1.1\%), and NF1 (1, 0.4\%) genes. Conclusions Monogenic mutations are not uncommon in young stroke patients. Further investigation with larger sample size might provide more precise information on the prevalence of stroke related monogenic disorders in young-age Korean stroke population.

Contact Email: gooday19@gmail.com

\section{Interleukin-6 signaling effects on ischemic stroke and cardiovascular disease: A Mendelian randomization study}

\author{
Marios K. Georgakis, $M D^{1,2}$, Rainer Malik, $P h D^{1}$, Dipender Gill, \\ $M D^{3}$, Martin Dichgans, $M D^{1,4,5}$
}

Objective To explore whether downregulated interleukin-6 (IL-6) signaling is associated with the risk of ischemic stroke and its subtypes, as well as with other cardiovascular phenotypes. Background Studies in humans and experimental models suggest that interfering with IL-6 signaling might lower risk of coronary artery disease. However, there is only limited evidence that such an approach would also be effective for ischemic stroke. Design/Methods In a genome-wide association study of 204,402 European individuals, we identified genetic proxies for downregulated IL- 6 signaling by selecting variants in the gene encoding IL-6 receptor (IL-6R) that were associated with lower C-reactive protein (CRP) levels, a downstream effector of IL-6 signaling. We then applied twosample Mendelian Randomization (MR) to explore associations with ischemic stroke and its major subtypes (large artery stroke, cardioembolic stroke, small vessel stroke) in the MEGASTROKE dataset (34,217 cases and 404,630 controls), with coronary artery disease in the CARDIoGRAMplusC4D dataset $(60,801$ cases and 184,305 control), and with other cardiovascular disease phenotypes. Results We identified 7 genetic variants as proxies for downregulated IL-6 signaling, which showed consistent effects on upstream regulators (IL-6, soluble IL-6R) and downstream effectors (CRP, fibrinogen) of the IL-6 signaling as the pharmacological blockade of IL6R. In MR, proxies for downregulated IL-6 signaling were associated with lower risk of ischemic stroke (OR scaled to 
a CRP-lowering effect of IL-6R pharmacological blockade: $0.89,95 \%$ CI: $0.82-0.96$ ) and coronary artery disease (OR: 0.84, 95\% CI: 0.77-0.90). Focusing on ischemic stroke subtypes, we found significant associations with risk of large artery (OR: $0.76,95 \%$ CI: $0.62-0.93$ ) and small vessel stroke (OR: 0.71, 95\% CI: 0.59-0.86), but not cardioembolic stroke (OR: 0.95, 95\% CI: 0.74-1.22). Proxies for IL-6 signaling inhibition were further associated with lower risks of myocardial infarction, aortic aneurysm, atrial fibrillation, and carotid plaque. Conclusions We provide evidence for a causal effect of IL-6 signaling on ischemic stroke, coronary artery disease, and related cardiovascular phenotypes. IL-6R blockade might represent a valid therapeutic target for lowering risk of ischemic stroke and cardiovascular risk in general and should thus be investigated in clinical trials.

Contact Email: marios.georgakis@med.uni-muenchen.de

\section{Circulating monocyte} chemoattractant protein- 1 is associated with risk of incident stroke: a meta-analysis of population-

\section{based studies}

Marios K. Georgakis, $\mathrm{MD}, \mathrm{MSc}^{1,2}$, Rainer Malik, $\mathrm{PhD}^{1}$, Harry Björkbacka, PhD ${ }^{3}$, Tiberiu Alexandru Pana, BSc ${ }^{4}$, Serkalem Demissie, PhD ${ }^{5,6}$, Colby Ayers, MS ${ }^{7}$, Mohamed A. Elhadad, $M S c^{8,9,10}$, Myriam Fornage, $P h D^{11}$, Alexa Beiser, $P h D^{5,6,12}$, Emelia J. Benjamin, $M D, S c M^{6,13,14}$, S. Matthijs Boekholdt, MD, $P h D^{15}$, Gunnar Engström, $M D, P h D^{3}$, Christian Herder, $P h D^{16,17,18,}$ Ron C. Hoogeveen, $P h D^{19}$, Wolfgang Koenig, $M D^{10,20,21}$, Olle Melander, $M D, P h D^{3}$, Marju Orho-Melander, $P h D^{3}$, Alexandru Schiopu, $M D, P h D^{3,22}$, Martin Söderholm, $M D, P h D^{3}$, Nick Wareham, $P h D^{23}$, Christie M. Ballantyne, $M D^{19}$, Annette Peters, $P h D^{9}$, Sudha Seshadri, $M D^{6,13,24}$, Phyo K. Myint, $M D^{4}$, Jan Nilsson, $M D, P h D^{3}$, James A. de Lemos, $M D^{7}$, and Martin Dichgans, $M D^{1,25,26}$

Objective To determine whether circulating levels of MCP1 are associated with risk of incident stroke in the general population. Background Pro-inflammatory cytokines have been identified as potential targets for lowering vascular risk. Experimental evidence and Mendelian randomization suggest a role of monocyte-chemoattractant protein-1 (MCP-1) in atherosclerosis and stroke. However, data from large-scale observational studies are lacking. Design/Methods We used previously unpublished data on 17,180 stroke-free individuals (mean age $56.7 \pm 8.1$ years; $48.8 \%$ males) from six population-based prospective cohort studies and explored associations between baseline circulating MCP-1 levels and risk of any stroke, ischemic stroke, and hemorrhagic stroke over a mean follow-up interval of 16.3 years (280,522 person-years at risk; 1,435 incident stroke events). We applied Cox proportional hazard models and pooled hazard ratios (HR) using random-effects meta-analyses. Results Following adjustments for age, sex, race, and vascular risk factors, higher MCP-1 levels were associated with increased risk of any stroke (HR per 1 SD increment in ln-transformed MCP-1: 1.07, 95\% CI: 1.01-1.14). Focusing on stroke subtypes, we found a significant association between baseline MCP-1 levels and higher risk of ischemic stroke (HR: $1.11,[1.02-1.21]$ ), but not hemorrhagic stroke (HR: 1.02, [0.82-1.29]). The results followed a dose-response pattern with a higher risk of ischemic stroke among individuals in the upper quartiles of MCP-1 levels as compared to the 1st quartile (HRs: 2 nd quartile: 1.19 [1.00-1.42]; 3rd quartile: 1.35 , [1.14-1.59]; 4th quartile: $1.38,[1.07-1.77])$. There was no indication for heterogeneity across studies and in a sub-sample of four studies (12,516 individuals) the risk estimates were stable after additional adjustments for circulating levels of interleukin-6 and high-sensitivity $\mathrm{C}$-reactive protein. Conclusions Higher circulating levels of MCP-1 are associated with increased long-term risk of stroke. Our findings along with genetic and experimental evidence suggest that MCP-1-signaling might represent a therapeutic target to lower stroke risk.

Contact Email: marios.georgakis@med.uni-muenchen.de

\section{Genome wide association study of stroke in Indian population}

Kameshwar Prasad, MD, MMSc ${ }^{1}$, Amit Kumar, $P h D, M P H^{1}$, Ram Sagar, $P h D^{1}$, Deepti Vibha, MD, DM, MBBS ${ }^{1}$, Achal Srivastava, $M D, D M, M B B S^{1}$, Subbiah Vivekanandhan, $P h D^{1}$, Afza Akbar, $M S^{1}$, Ajay Garg, $M D^{2}$, Arti Gulati, $M B B S^{3}$, Bhavna Kaul, MD, DM ${ }^{4}$, Neera Chaudhry, MD, MBBS ${ }^{4}$, Samudrala Raghavan ${ }^{4}$, Sankar Prasad Gorthi ${ }^{5}$, Salil Gupta, Chandra Sekhar Agrawal ${ }^{6}$, Dheeraj Mohania ${ }^{6}$, Surekha Dabla ${ }^{7}$, Samander Kaushik, $\mathrm{PhD}, \mathrm{MSc}^{8}$, Rohtas Kanwar Yadav, $M D^{9}$, Kuljeet Singh Anand, $D M, M B^{10}$, Shriram Sharma, $D M^{11}$, P.N. Sylaja, $M D^{12}$, and Pankaj Sharma, $M D, P h D^{13}$

Objective To decipher genetic susceptibility loci for occurrence of stroke in Indian population using Genome wide genotyping approach. Background The genetics of stroke is currently receiving worldwide attention for the development of precision medicine. The genes underlying the risk of stroke in Indian population remains undetermined. Design/ Methods In a multi-centric hospital-based case-control study, eight teaching hospitals recruited cases and controls from different parts of India. Eligibility criteria included age $18-85$ years, both sexes with history of stroke ever in life, Indian ancestry, and absence of any major neurological disorders. After obtaining written informed consent of patients/proxy, venous blood samples $(6 \mathrm{~mL})$ were collected in specific EDTA coated vials for DNA isolation using phenol-chloroform method and stored at $-80^{\circ} \mathrm{C}$ at 
coordinating centre. The genotyping of these samples is ongoing using Global Screening Array Illumina chip which consists of more than 0.6 million markers. The process is divided into two phases: Discovery phase (1,500 cases and 2,500 controls) and Replication Phase. The current abstract refers only to discovery phase of 480 samples genotyped so far. The association analysis was performed using PLINK v1.9. Results A total of 480 samples consisting of 759,993 variants were analyzed using GWAS approach. Quality control parameters for the present study for filtering SNPs and samples for analysis were applied separately for both stroke cases and control subjects. Samples with low call rate $(<95 \%)$, sex discrepancies were removed. We also removed SNPs within regions of known high linkage disequilibrium (LD) and with minor allele frequency $<0.05$. After completing this $\mathrm{QC}$ a total of 468 samples were used for the final analysis. HWE threshold were set at $<1 \mathrm{e}-06$ for controls and $<1 \mathrm{e}-10$ for cases. Markers at high LD region were pruned. Subsequently, 453,046 makers from 460 samples (Case 270 and Controls 190) were selected for association analysis. A total of 241 markers were observed to be significantly associated with ischemic stroke $\left(p<5 \times 10^{-7}\right)$. Among 241 identified markers, 73 were rare variants (having frequency $<1 \%$ in 1,000 Genome project). These were annotated and it was observed that the loci harboring in Phakophilin-2 (rs144620127), Ankyrin-2 (rs 370647591), Plexin- domain containing 1 (147007318), Carboxipeptidase A6 (rs 190589031) gene are having known association towards pathology of stroke. Conclusions The genetic locus on chromosome $12 \mathrm{p} 11$ was found to be associated with increased risk of stroke in this preliminary analysis. The locus on chromosome $12 \mathrm{p} 13$ has been widely reported and replicated in other GWAS studies. This is probably the first GWAS study of stroke from India. It may identify ethnicity specific genetic susceptible loci for Indian stroke patients.

Contact Email: kp0704@gmail.com

\section{Iron and cardiovascular disease: where we have got to with genetics}

\section{Dipender Gill, $M D^{1}$}

Objective To summarize where we have got to with using genetics to understand the effects of iron status on cardiovascular disease and highlight recent advances. Background Iron is integral to metabolism and healthy physiological function, and variations in its levels have been implicated in cardiovascular disease. Recent work has suggested that higher iron status may have a protective effect in atherosclerosis related to dyslipidemia, but a detrimental effect on thrombosis related to stasis of blood. ${ }^{1-4}$ Design/Methods This work performs Mendelian randomization mediation analyses to explore the protein mediators of the effect of iron status on disease outcomes. Results Using state-of-the-art statistical methods and large-scaled proetomic datasets, it is possible to delineate the causal mechanism relating iron status to health outcomes through protein mediators. Conclusions This works to offer insight into the mechanisms by which iron status affects health outcomes and also identifies potential protein targets for intervention. While applied to the paradigm of iron status related health outcomes, this approach may equally be transferred across other disease processes.

Contact Email: dipender.gill@imperial.ac.uk

\section{References}

1. Gill D, Brewer CF, et al. Effects of genetically determined iron status on risk of venous thromboembolism and carotid atherosclerotic disease: a Mendelian Randomization Study. J Am Heart Assoc 2019;8(15):e012994.

2. Gill D, Benyamin B, et al. Associations of genetically determined iron status across the phenome: A mendelian randomization study. PLoS Med 2019;16(6): e1002833.

3. Gill D, Monori G, et al. Iron status and risk of stroke. Stroke 2018;49(12):2815-2821.

4. Gill D, Del Greco MF, et al. The effect of iron status on risk of coronary artery disease: a Mendelian Randomization Study. Arterioscler Thromb Vasc Biol 2017;37(9): 1788-1792.

\section{Increased mean transit time and blood brain permeability in}

\section{asymptomatic white matter lesions of ischemic origin}

Blake E. Dewey, $B A^{1}$, Xiang $X u, P h D^{1}$, Linda Knutsson, $P h D$, $M \mathrm{Mc}^{1}$, Amod Jog, $P h D^{2}$, Jerry L. Prince, $P h D^{2}$, Peter B. Barker, DPhil ${ }^{1}$, Peter C.M. van Zijl, $P h D^{1}$, Richard Leigh, $M D^{1}$, and Paul Nyquist, $M D, M P H^{1}$

Objective To determine Meat transit time and Blood brain permeability with in ischemic white matter lesions in asymptomatic people. Background White matter (WM) lesions of presumed ischemic origin have been associated with increased risk of stroke and cognitive and motor decline and are a subject of public health research. Engineering new MRI pipelines allowing for determination of mean transit time (MTT) and blood brain barrier permeability (BBBP) within WM lesions is required for long-term populationbased studies of lesion progression in patients with dementia and vascular cognitive impairment. Design/Methods WM lesion volumes in 24 asymptomatic individuals were determined using an automated segmentation methodology, S3DL, with manual correction to remove false positives. A double contrast injection scheme was used to measure both Ktrans using dynamic contrast enhanced (DCE) imaging and $\mathrm{K} 2$ using dynamic susceptibility contrast (DSC) imaging which also provided perfusion-related measures. BBBP was measured as K2 within segmented WM lesions and compared with normal white and gray matter. Results The mean transit time (MTT) was found to be significantly prolonged $(8.11, p<0.001$ Wilcoxon Signed-Rank Test) in WM lesions when compared to normal appearing white and gray matter. There was no significant difference in DCE-Ktrans (0.018, 
$p=0.351)$ between the lesions and the white/gray matter. Permeability measured in the WM lesions using the DSC-K2 method was increased and was correlated with increasing total WM lesion volume (Spearman correlation 0.44; $p<$ 0.046). Conclusions In this first study using an advanced WM lesion automated segmentation pipeline, we measured DCE and DSC perfusion and permeability variables within WM lesions and compared them to normal white and grey matter in healthy people. We observed increasing MTT, within WM lesions as compared to unaffected white and gray matter. Using the DSC-K2 method, BBBP was higher within WM lesions in these asymptomatic people and correlated with increasing total lesion volume.

Contact Email: pnyquis1@jhmi.edu

\section{Homocysteine, choline and lipids are associated with severe extracranial carotid artery stenosis: A metabolomics study}

C.K. Cheng, $M D^{1,2}$, M.L. Cheng, $P h D^{3,4}$, M.S. Shiao, $P h D^{3,4}$, C.N. Lin, PhD ${ }^{2,5}$, and T.H. Lee, $M D, P h D^{1,5}$

Objective To investigate if metabolites may also act as biomarkers for carotid artery stenosis. Background Significant genetic association has been found in patients with severe carotid artery stenosis (CAS). The present study wished to investigate if metabolites may also act as biomarkers for CAS. Design/Methods Consecutive patients with at least one carotid artery stenosis more than $60 \%$ on cerebral angiography were prospectively recruited from May 2007 to January 2016. Normal controls were recruited from outpatient clinic who had no stroke and coronary artery disease (CAD) history, and the brain magnetic resonance or computed tomographic angiography showed bilateral CAS less than 30\%. Risk factor profile, clinical characteristics, age, and clinical features were recorded. All subjects were male, and none had diabetes. 1H-NMR spectroscopy-based metabolomics analysis was carried out for plasma samples. Results Totally, 130 male subjects were recruited. Age had no significant difference between the controls and CAS group $(60.2 \pm 5.9$ vs $63.3 \pm 6.0, p=0.050)$. The CAS group had significantly higher frequency of $\mathrm{CAD}$, hypertension, smoking and alcohol but lower body mass index than the controls $(p<0.05)$. The laboratory tests showed CAS group had significantly higher level of homocysteine but lower levels of cholesterol, high-density lipoprotein and hemoglobin than the controls $(p<0.05)$. The 1H-NMR based plasma metabolomics analysis indicated that choline was significantly lower in CAS patients. The VIP values of lipids were greater than 1.0, which were considered significantly different. Conclusions Our results suggest homocysteine, choline and lipids in association with traditional risk factors may be involved in the pathogenesis of CAS. Diet adjustment to control homocysteine, choline and lipids may be helpful for the prevention of CAS.

Contact Email: neurockoung@gmail.com

\section{Characterization of polygenic risk in early onset stroke cases by comparison to later onset stroke}

Thomas J. Jaworek, BS $S^{1}$ Brady J. Gaynor, $M S^{1}$, Kathleen Ryan, $M P H^{1}$, Patrick F. McArdle, $P h D^{1}$, John W. Cole, $M D^{1}$, Steven J. Kittner, $M D^{1}$, Huichun $X u, M D, P h D^{1}$, and Braxton $D$. Mitchell, $P h D^{1}$; for the SiGN Network and Early Onset Ischemic Stroke Consortium

Objective Determine the contribution of common variation to early onset ischemic stroke via known stroke risk factors using the largest sample of early onset cases to date ( $\mathrm{n}=$ 5,847 ). The goal of this study is to characterize whether the genetic contribution of known stroke risk factors is similar in early-onset stroke and older-onset cases. Background The extent to which common variation in known risk factors contributes to early-onset ischemic stroke risk remains unclear. Polygenic risk score analysis can be used to identify common genetic etiologies between stroke risk factors and stroke as an outcome. Design/Methods We performed a polygenic risk score analysis with 5,847 IS early-onset cases under age $60,15 \mathrm{~K}$ later-onset cases, and $32 \mathrm{~K}$ controls. Using publicly available GWAS summary statistics, we calculated PRSs for known stroke risk factors, including diabetes, blood pressure, migraine, lipid-related traits, and coagulation factors. We used logistic regression to test the association with these scores and all ischemic stroke. We then compared the effect size of these associations using a Wald test. Results We found associations between T2D, SBP, DBP, and hypertension risk scores in both early and late onset cases. We also observed associations with BMI and total cholesterol only in early onset cases. The effect size for the T2D PRS was greater in early onset cases compared to late onset ( $p=$ 0.006). In the coagulation factors we tested, we found associations with FXI and stroke in both early and late onset cases, but only found an association with VWF and F8 in the early onset cases $\left(p=2.93 \times 10^{-07}, p=1.23 \times 10^{-06}\right.$ respectively). Conclusions We observed differences in the polygenic risk profiles between early-onset and later-onset stroke. Our associations with coagulation factor scores are consistent with previous findings linking coagulation to early onset stroke. These differences may be important determinants of the age of stroke occurrence.

Contact Email: tjaworek@som.umaryland.edu 


\section{Automated classification of clinical} MRI stroke datasets with a recurrent convolutional neural network

Y. $L i$, $P h D^{1}$, B. Hancock, $B S c^{1}, M$. Etherton, $M D, P h D^{2}$, T. Hoang ${ }^{1}$, S. Mocking, $M S^{1}$, E. McIntosh, $B A^{1}$, R. Irie, $P h D^{1}$, M. Bouts, $P h D^{1}$, J. Broderick, $M D^{3}$, J.W. Cole, $M D^{4}$, K. Donahue, $B S^{2}, A$. Giese, $M D^{2}$, E. Giralt-Steinhauer, $M D, P h D^{5}$, J. JimenezConde, $M D, P h D^{5}$, C. Jern, $M D$, $P h D^{6}$, S. Kittner, $M D, M P H^{4}$, D. Kleindorfer, $M D^{3}$, R. Lemmens, $M D, P h D^{7}, P . M c A r d l e, P h D^{8}$, J. Meschia, $M D^{9}$, A. Lindgren, $M D, P h D^{10,11}$, J. Rosand, $M D^{2,12}$, T. Rundek, $M D, P h D^{13}$, R. Sacco, $M D, M S^{13}$, M. Schirmer, $P h D^{2}$,
R. Schmidt, $M D^{14}$, P. Sharma, $M D, P h D^{15}$, A. Slowik, $M D, P h D^{16}$, V. Thijs, $M D$, $P h D^{17}$, J. Wasselius, $M D, P h D^{11}$, B. Worrall, $M D$, $M \mathrm{Mc}^{18}, \mathrm{~N}$. Rost, $\mathrm{MD}, \mathrm{MPH} \mathrm{H}^{2}$, and $\mathrm{O} . \mathrm{Wu}, \mathrm{PhD}^{1}$

Objective To develop an algorithm that will automate the classification of clinical MRI series. Background Fundamental advances in stroke care will require pooling imaging phenotype data from multiple centers, to complement the current aggregation of genomic, environmental, and clinical information. Sharing clinically acquired MRI data from multiple hospitals is challenging due to inherent heterogeneity of clinical data, where the same MRI series may be labeled differently depending on vendor and hospital.

Table 1 (Abstract 29)

Table: Number of available series for each cohort

and category

\begin{tabular}{|c|c|c|c|}
\hline \multirow{2}{*}{ Modality } & \multicolumn{3}{|c|}{ Data set } \\
\hline & Training & Validation & Testing \\
\hline FLAIR & 768 & 92 & 113 \\
\hline $\begin{array}{l}\text { Gradient Echo Susceptibility } \\
\text { Image }\end{array}$ & 339 & 39 & 78 \\
\hline Proton Density & 146 & 18 & 0 \\
\hline T1-weighted Image & 975 & 103 & 126 \\
\hline $\begin{array}{l}\text { Post-contrast T1-weighted } \\
\text { Image }\end{array}$ & 470 & 65 & 32 \\
\hline T2-weighted image & 1031 & 124 & 97 \\
\hline DWI & 374 & 43 & 104 \\
\hline $\begin{array}{l}\text { Apparent Diffusion } \\
\text { Coefficient Image }\end{array}$ & 563 & 60 & 105 \\
\hline Exponential Image & 164 & 24 & 98 \\
\hline b0 map & 121 & 15 & 54 \\
\hline $\begin{array}{l}\text { Diffusion Tensor Imaging } \\
\text { raw data }\end{array}$ & 270 & 36 & 100 \\
\hline Fractional Anisotropy (FA) & 125 & 20 & 104 \\
\hline FA color & 62 & 9 & 43 \\
\hline $\begin{array}{l}\text { Dynamic Susceptibility } \\
\text { Contrast (DSC) perfusion } \\
\text { weighted imaging raw data }\end{array}$ & 71 & 11 & 41 \\
\hline DSC post-processed maps & 422 & 63 & 259 \\
\hline $\begin{array}{l}\text { DSC color post processed } \\
\text { maps }\end{array}$ & 56 & 12 & 0 \\
\hline $\begin{array}{l}\text { MR Angiograms (MRA) raw } \\
\text { Images }\end{array}$ & 589 & 69 & 14 \\
\hline MRA post-processed maps & 3066 & 337 & 585 \\
\hline MRA timing data & 77 & 3 & 13 \\
\hline Localizer & 688 & 81 & 131 \\
\hline $\begin{array}{l}\text { Multi-volume series: } \\
\text { MRA/MRA raw data }\end{array}$ & 381 & 33 & 162 \\
\hline $\begin{array}{l}\text { Multi-volume series: } \\
\text { DWI/b0 }\end{array}$ & 276 & 26 & 7 \\
\hline Multi-volume series: PD/T2 & 135 & 21 & 0 \\
\hline Other categories & 90 & 14 & 51 \\
\hline
\end{tabular}


Furthermore, the de-identification process may remove data describing the MRI series, requiring human review. However, manually annotating the MRI series is not only laborious and slow but prone to human error. Design/Methods We randomly selected 1,000 subjects from the MRIGENetics Interface Exploration study and partitioned them into 800 training, 100 validation and 100 testing subjects. We categorized the MRI series into 24 groups (table 1). The RCNN used a modified AlexNet to extract features from $2 \mathrm{D}$ slices. AlexNet was pretrained on ImageNet photographs. Since clinical MRI are 3D and 4D, a gated recurrent unit neural network was used to aggregate information from multiple 2D slices to make the final prediction. Results We achieved a classification accuracy (correct/total cases) of $99.8 \%, 98.5 \%$ and $97.5 \%$ on the training, validation and testing set, respectively. The averaged F1-score (percent overlap between predicted cases and actual cases) over all categories were $99.8 \% 98.2 \%$ and $94.4 \%$ on the training, validation and testing set. Conclusions In conclusion, we showed that automated annotation of MRI series by repurposing deep-learning techniques used for photographic image recognition tasks is feasible. Such methods can be used to facilitate high throughput curation of MRI data acquired across multiple centers and enable scientifically productive collaboration by researchers and, ultimately enhancing big data stroke research.

Contact Email: ona@nmr.mgh.harvard.edu

\section{Big data approaches to} neuroimaging: An automated processing pipeline to extract cerebral edema phenotypes from serial CT scans of stroke patients

Hossein Mohammadian Foroushani, $M S c^{1}$, Yasheng Chen, $P h D^{2}$, Ali Hamzehloo, $M D^{2}$, Jin-Moo Lee, $M D, P h D^{2}$, Daniel S. Marcus, $P h D^{3}$, and Rajat Dhar, $M D, F R C P C^{2}$
Objective Demonstrate feasibility of automated extraction of CSF volumes from serial CT scans of patients with acute ischemic stroke using an XNAT-based imaging platform and containerized-processing modules. Background Application of big data approaches holds great promise in uncovering new pathways and targets relevant to stroke pathophysiology. Cerebral edema is a major complication of stroke that would benefit from such biologic interrogation. We have proposed that measuring change in CSF volume from baseline to 24-hour computed tomography (CT) can serve as a quantitative endophenotype that reflects progression of cerebral edema after stroke. However, implementing such reverse translational studies would requires the integration of genomic data with imaging phenotypes. We have developed a platform (Stroke Neuro-Imaging Phenotype Repository, SNIPR) capable of archiving and processing serial CTs scans to extract this edema-related phenotype. Design/Methods SNIPR allows centralizing archiving of clinical brain imaging from multiple sites including direct transfer of images from clinical PACS. We have built a containerized (Docker-based) processing pipeline that can extract CSF volume from serial CT scans of patients with CT scans in the repository. This includes: (1) detection of axial brain sequences within a complex imaging session with multiple scans (using a neural network); (2) conversion of DICOM files into single NIfTI (Neuroimaging Informatics Technology Initiative) file for analysis; (3) selection and quality checks of which scan to process (e.g., adequate brain coverage, proper slice thickness); (4) preregistration for skull stripping; (5) registration of serial CTs to baseline and to brain atlas; (6) segmentation of CSF using U-net deep learning network. This seamless stroke edema pipeline was tested on 100 subjects to demonstrate feasibility. Results Imaging data from over 1,500 acute stroke patients have been anonymized and archived in SNIPR to date. Results from the feasibility testing demonstrated $90 \%$ success with some scans failing registration due to low scan quality. Registration of cranial volumes was accurate when these outliers were exlcuded. Moreover, CSF volumes obtained from the pipeline closely mirrored volumes obtained from prior non-pipeline-based segmentation. Conclusions We have been able to develop an image processing pipeline that can extract CSF volumes from $\mathrm{CT}$ scans

Figure 6 (Abstract 30) Outline of image processing pipeline to analyze CSF volumes from large cohorts of stroke patients

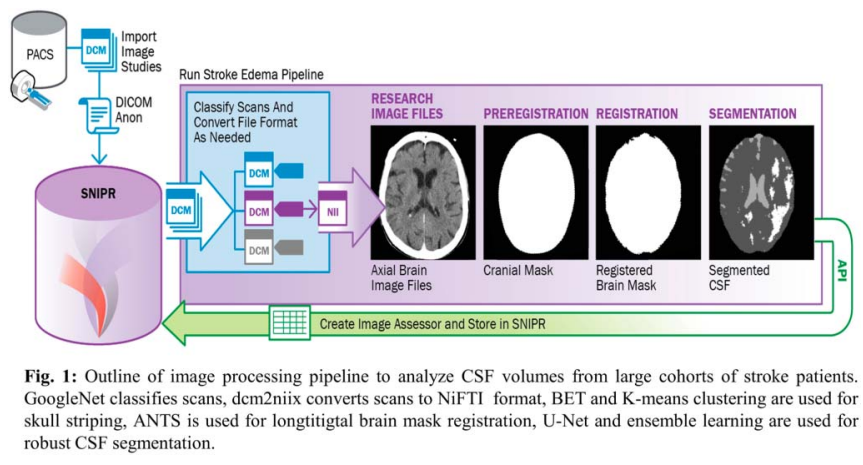


Table 2 (Abstract 31) Comparison of 3 tissue-based collateral flow metrics, with HIR demonstrating high correlation to all vascular measures of collateral flow and clinical outcome metrics

\begin{tabular}{|l|c|c|c|c|c|c|}
\hline & \multicolumn{2}{|c|}{ HIR } & \multicolumn{2}{c|}{ rC8V } & \multicolumn{2}{c|}{ PCI } \\
\hline & $\mathrm{R}^{2}$ & $\mathrm{p}-$ value & $\mathrm{R}^{2}$ & $\mathrm{p}$-value & $\mathrm{R}^{2}$ & $\mathrm{p}$-value \\
\hline Tan Scale & 0.43 & $1.2 \mathrm{E}-6^{* *}$ & 0.31 & $6.2 \mathrm{E}-5^{* *}$ & -0.02 & 0.61 \\
\hline $\begin{array}{l}\text { MCA flow } \\
\text { ratio }\end{array}$ & 0.34 & $1.1 \mathrm{E}-4^{* *}$ & 0 & 0.34 & -0.01 & 0.42 \\
\hline $\begin{array}{l}\text { MCA flow } \\
\text { delay }\end{array}$ & 0.26 & $8.6 \mathrm{E}-4^{* *}$ & -0.02 & 0.69 & -0.03 & 0.78 \\
\hline $\begin{array}{l}\text { 4-vessel M2 } \\
\text { delay }\end{array}$ & 0.22 & $4.3 \mathrm{E}-3^{* *}$ & -0.03 & 0.79 & -0.03 & 0.77 \\
\hline $\begin{array}{l}\text { MCA flow } \\
\text { dispersion }\end{array}$ & 0.13 & $0.02^{*}$ & 0.01 & 0.25 & 0 & 0.35 \\
\hline ANIHSS & 0.09 & $0.03^{*}$ & -0.01 & 0.48 & -0.02 & 0.80 \\
\hline 90-day mRS & 0.47 & $5.4 \mathrm{E} \cdot 0.5^{* *}$ & 0.31 & $1.6 \mathrm{E}-3^{* *}$ & -0.01 & 0.37 \\
\hline
\end{tabular}

- denotes $p<0.05, \cdots$ denotes $p<0.01$

of stroke patients in an automated high-throughput manner. Refinements in quality control and registration are still required, but this approach holds promise in obtaining edema-related endophenotypes on thousands of stroke patients, facilitating genomic studies of this important stroke-related complication (figure 6).

Contact Email: dharr@wustl.edu

\section{HIR outperforms $\mathrm{rCBV}$ and PCI as a tissue-based metric of collateral blood flow in acute ischemic stroke}

J.J. Derakhshan, $M D^{1}$, Z. Vardar, $M D^{1}$, A. Abedini, DPhil, M.S. Goyal, $M D, M S c^{1}$, R.C. McKinstry, $M D, P h D^{1}$, J.M. Lee $M D, P h D^{1}$

Objective To determine the best tissue-based metric of collateral blood flow after acute ischemic stroke (AIS). Background To perform large genetic studies of collateral flow after AIS, quantitative tissue-based metrics will be required. Three such metrics have been described: the hypoperfusion intensity ratio (HIR), relative cerebral blood volume (rCBV) and perfusion collateral index (PCI). While all of these metrics have been shown to correlate with collateral vessels via CTA and conventional angiography, all three have yet to be compared to each other. Design/Methods Semi-automated perfusion analysis software was written in Matlab (Mathworks, Natick, MA) and used to analyze CTP datasets in 43 patients with anterior circulation acute ischemic stroke. CTP tissue-based metrics ( $\mathrm{HIR}, \mathrm{rCBV}, \mathrm{PCI}$ ) were correlated to established vascular metrics (Tan scale, 4-vessel M2 delay) as well as novel MCA flow analyses (delay, dispersion and flow ratio of total iodine in the MCA territories) of collateral flow. In addition, tissue-based collateral flow metrics were correlated with clinical assessments, including $\triangle$ NIHSS (change in NIH Stroke Scale between 6 and 24 hours after onset), and 90 days modified Rankin Scale (mRS). T-tests and linear regression were performed in Matlab. Correlation analysis was performed in SPSS (IBM Corp, Armonk, NY). Correlations from the three tissue- based metrics were directly compared to one another. Results Of the three tissue-based collateral flow metrics, only HIR showed high correlation to all vascular measures of collateral flow (table 2). Moreover, HIR showed consistent correlation with both $\Delta$ NIHSS as well as 90 days mRS (table 2). Conclusions HIR is the tissue-based collateral flow metric that most consistently correlated to multiple vascular collateral flow metrics as well as clinical outcome metrics, suggesting its relative superiority to $\mathrm{rCBV}$ and PCI.

Contact Email: jamald@wustl.edu

\section{A deep learning algorithm replicates expert human white matter hyperintensity segmentation better than other human raters}

Yasheng Chen, PhD ${ }^{1, *}$, Chia-Ling Phuah, MD, MMSc ${ }^{1, *}$, Jeremy Strain, PhD ${ }^{2, *}$, Collin Kilgore ${ }^{2}$, Hongyu An, $P h D^{2}$, Beau Ances, $M D, P h D, M S c^{1}+$, and Jin-Moo Lee, $M D, P h D^{1,}+$; for the $A l z-$ heimer's Disease Neuroimaging Initiative

Objective Accurate and reliable WMH segmentation from MR images are therefore prerequisites for large-scale studies for understanding $\mathrm{WMH}$ pathogenesis and disease progression. We compare the performance of deep learning (DL) based WMH segmentation within the context of human inter-rater variability. Background White matter hyperintensities (WMH) on T2-weighted FLAIR magnetic resonance (MR) images are prevalent imaging findings in cerebral small vessel disease (SVD) and normal aging, and are associated with increased risks of cognitive impairment, stroke and dementia. However, interpretation by different human raters contribute to the low reproducibility of WMH segmentation across cohorts. This confounds comparability across studies and limit their power to detect and follow the progression of WMHs. Accurate and reliable WMH segmentation from MR images are therefore prerequisites for large-scale studies for understanding WMH 
Figure 7 (Abstract 32) Results of Deep Learning algorithm achieving higher correlation compared with interrater correlation for both voxel-wise (A) and volumetric WMH agreements (B)
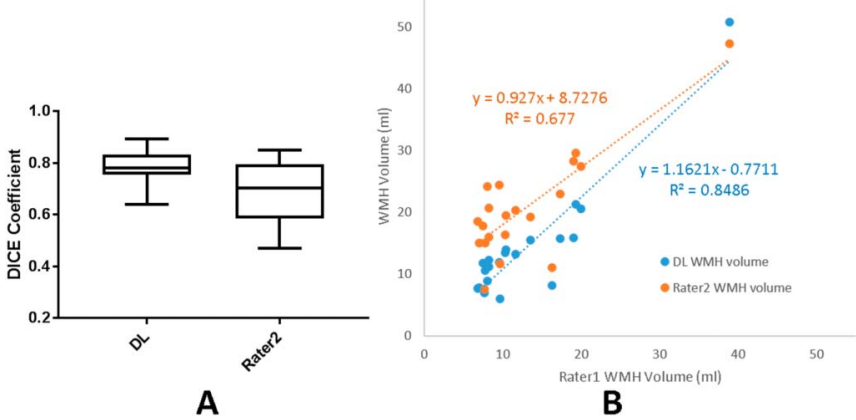

pathogenesis and disease progression. Here, we compare the performance of deep learning (DL) based WMH segmentation within the context of human inter-rater variability. Design/ Methods MRIs used in this study were obtained from the Alzheimer's Disease Neuroimaging Initiative (ADNI) public database. We randomly selected MR FLAIR images from 50 ADNI participants (cognitively normal, MCI, and dementia). Two expert human raters independently performed WMH segmentation of the dataset after aligning WMH definitions with further consensus from two neuroradiologists: Observer 1 produced manual delineation of WMH for all 50 images; Observer 2 generated WMH segmentation masks in a subset of 20 participants semi-automatically using an inhouse MATLAB program, individually thresholding (1.2 SD contrast intensity) each WMH cluster in the original FLAIR images followed by manual correction. Using the WMH masks delineated by Observer 1 as "ground truth," we performed 5-fold cross validation across the dataset, where 40 images are used as training samples, and 10 images are used for testing a DL neural network based on a modified version of the U-net architecture for automated WMH segmentation. The selection of images for training and testing in each cross validation was done randomly. WMH segmentation accuracy was evaluated using dice similarity coefficient (DSC) and volume correlation: (1) comparing agreement between DL segmentation results and WMH segmentation masks produced manually by Observer 1; (2) comparing interrater reliability between WMH segmentation masks produced manually by Observer 1 and Observer 2 . Results Our DL algorithm achieved higher ranges of correlation coefficients compared with interrater correlation coefficients for both voxel-wise (DSC $=0.786 \pm 0.064$ vs $0.685 \pm$ $0.116 ; p=0.003$, paired $t$-test; figure $7 \mathrm{~A}$ ) and volumetric WMH agreements $\left(R^{2}=0.849\right.$ vs 0.677 ; figure $\left.7 \mathrm{~B}\right)$. Conclusions We have demonstrated that a deep learning approach is able to achieve more reproducible WMH quantification by eliminating variability caused by userdependent bias from subjective manual lesion identification and quantification from human expert raters, even after formal alignment of rater training.

Contact Email: chen.yasheng@gmail.com

\section{GENISIS GWAS reveals two loci that implicate excitotoxicity in ischemic brain injury}

Laura Heitsch, $M D^{1, *}$, Laura Ibanez, $P h D^{1, *}$, Caty Carrera, $M D^{2}$, Daniel Strbian, $M D, P h D^{3}$, Agnieszka Slowik, $M D, P h D^{4}$, Antonio Arauz, $M D, P h D^{5}$, Israel Fernandez, $P h D^{2}$, Joan Montaner, $\mathrm{MD}, \mathrm{PhD}^{2}$, Carlos Cruchaga, $\mathrm{PhD}^{1, *}$, and Jin-Moo Lee, $M D, P h D^{1, *}$

Objective To identify genetic loci related to early neurological changes after ischemic stroke using a genome wide association study (GWAS). Background Following acute ischemic stroke (AIS) onset, neurological deficits can be highly unstable within the first 24 hours. We hypothesized that this early change, defined here as $\Delta$ NIHSS24h (NIHSS baseline - NIHSS 24 hours), could serve as a quantitative phenotype to capture the genetic architecture of ischemic brain injury. Design/Methods AIS patients were prospectively enrolled between 2008 and 2019 at 7 sites (St Louis, Barcelona, Helsinki, Krakow, Korea, Costa Rica and Mexico). NIHSS scores were obtained within 6 hours and again at 24 hours after stroke onset. Genome-wide genotyping was generated for rare and common variants, imputing up to 6 million single nucleotide polymorphisms (SNPs) for all subjects $(\mathrm{N}=5,487)$. $\Delta$ NIHSS24h was used as a quantitative trait in an association model, with covariates that included baseline NIHSS, age, sex, PCA1, PCA2 and genotyping round. All samples were analyzed together using Plink 1.9. They are also analyzed by site and then, we used Mantra to perform a trans-ethnic meta-analysis. Results A GWAS for early stroke outcome $(\Delta$ NIHSS $=$ NIHSS6h - NIHSS24h $)$, and subsequent functional annotation analyses identified ADAM 23 and GRIA1 (see Ibanez et al) as genes associated with $\triangle$ NIHSS. These loci were associated with genes involved in regulating trans-synaptic excitability. ADAM23, a transmembrane protein involved in cell-cell interaction, is expressed in excitatory and inhibitory neurons in the brain, specifically on pre-synaptic membranes. It appears to play a role in bridging presynaptic to postsynaptic membranes and PSD-95 (post-synaptic density scaffold complex) via the linker protein LGI-1 (Leucine-rich, glioma 
inactivated 1). PSD-95, in turn, binds to a variety of neurotransmitter receptors, thereby bringing receptors in close apposition to the presynaptic neurotransmitter release machinery and regulating synaptic excitability. GRIAl encodes for the glutamate ionotropic receptor AMPA type Subunit 1. This glutamate receptor, one of the predominant excitatory neurotransmitter receptors in the mammalian brain, links to PSD-95 and regulates synaptic excitability. Conclusion Thus, these two loci support data from animal models that excitotoxicity is an important mechanism involved in human ischemic brain injury. In addition, these findings support the feasibility of using $\Delta$ NIHSS as a quantitative phenotype to discover novel targets for the treatment of AIS.

Contact Email: ibanezl@wustl.edu 


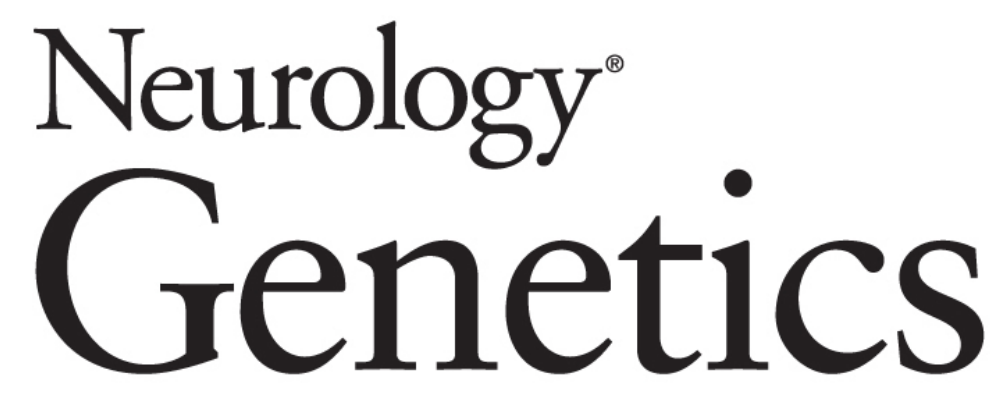

\section{Proceedings of the 26th International Stroke Genetics Consortium Workshop: St. Louis, MO \\ Jin-Moo Lee and Carlos Cruchaga \\ Neurol Genet 2020;6;e422 \\ DOI 10.1212/NXG.0000000000000422}

\section{This information is current as of May 21, 2020}

Updated Information \&

Services

References

Permissions \& Licensing

Reprints including high resolution figures, can be found at: http://ng.neurology.org/content/6/1_Supplement_1/e422.full.html

This article cites 4 articles, 1 of which you can access for free at: http://ng.neurology.org/content/6/1_Supplement_1/e422.full.html\#\#ref -list-1

Information about reproducing this article in parts (figures,tables) or in its entirety can be found online at:

http://ng.neurology.org/misc/about.xhtml\#permissions

Information about ordering reprints can be found online: http://ng.neurology.org/misc/addir.xhtml\#reprintsus

Neurol Genet is an official journal of the American Academy of Neurology. Published since April 2015, it is an open-access, online-only, continuous publication journal. Copyright ( 2020 American Academy of Neurology. All rights reserved. Online ISSN: 2376-7839.

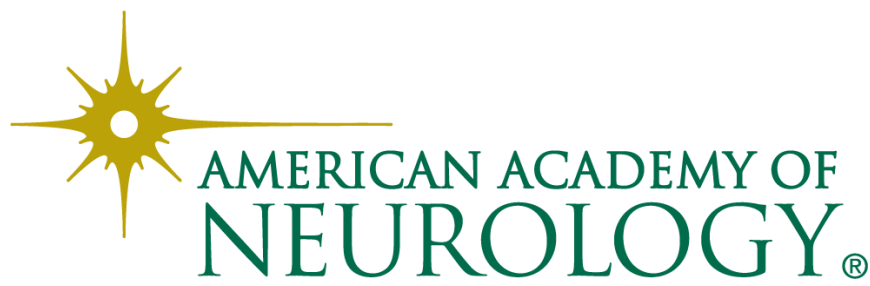

